\title{
RANDOM WALKS, ARRANGEMENTS, CELL COMPLEXES, GREEDOIDS, AND SELF-ORGANIZING LIBRARIES
}

\author{
ANDERS BJÖRNER
}

TO LÁSZLÓ LOVÁSZ ON HIS 60TH BIRTHDAY

\begin{abstract}
The starting point is the known fact that some much-studied random walks on permutations, such as the Tsetlin library, arise from walks on real hyperplane arrangements. This paper explores similar walks on complex hyperplane arrangements. This is achieved by involving certain cell complexes naturally associated with the arrangement. In a particular case this leads to walks on libraries with several shelves.

We also show that interval greedoids give rise to random walks belonging to the same general family. Members of this family of Markov chains, based on certain semigroups, have the property that all eigenvalues of the transition matrices are non-negative real and given by a simple combinatorial formula.

Background material needed for understanding the walks is reviewed in rather great detail.
\end{abstract}

\section{Contents}

1. Introduction

2. Real hyperplane arrangements

2.1 Basics

2.2 The braid arrangement

2.3 Cell complexes and zonotopes

2.4 The permutohedron and the $k$-equal arrangements

3. Complex hyperplane arrangements

3.1 Basics

3.2 Cell complexes

3.3 Complexified $\mathbb{R}$-arrangements

4. Random walks

4.1 Walks on semigroups

4.2 Walks on $\mathbb{R}$-arrangements

4.3 Walks on $\mathbb{C}$-arrangements

4.4 Walks on libraries

4.5 Walks on greedoids

5. Appendix

5.1 A generalized Zaslavsky formula

5.2 Lattice of intervals

5.3 Interval greedoids 


\section{INTRODUCTION}

The following random walk, called Tsetlin's library, is a classic in the theory of combinatorial Markov chains. Consider books labeled by the integers $1,2, \ldots, n$ standing on a shelf in some order. A book is withdrawn according to some probability distribution $w$ and then placed at the beginning of the shelf. Then another book is withdrawn according to $w$ and placed at the beginning of the shelf, and so on. This Markov chain is of interest also for computer science, where it goes under names such as dynamic file management and cache management.

Much is known about the Tsetlin library, for instance good descriptions of its stationary distribution, good estimastes of the rate of convergence to stationarity, exact formulas for the eigenvalues of its transition matrix $P_{w}$, and more. These eigenvalues are nonnegative real and their indexing and multiplicities, as well as their value, are given by very explicit combinatorial data.

The Tsetlin library is the simplest of a class of Markov chains on permutations that can be described in terms of books on a shelf. Instead of one customer visiting the library to borrow one book which when returned is placed at the beginning of the shelf, we can picture several customers who each borrows several books. When the books are returned, the books of the first borrower are placed at the beginning of the shelf in the induced order (i.e. the order they had before being borrowed). Then the books of the second borrower are placed in their induced order, and so on. Finally, the remaining books that noone borrowed stand, in the induced order, at the end of the shelf.

The analysis of such a "dynamic library" became part of a vastly more general theory through the work of Bidigare, Hanlon and Rockmore [2], continued and expanded by Brown and Diaconis [13, 14, 15, 16]. They created an attractive theory of random walks on hyperplane arrangements $\mathcal{A}$ in $\mathbb{R}^{d}$, for which the states of the Markov chain are the regions making up the complement of $\cup \mathcal{A}$ in $\mathbb{R}^{d}$. When specialized to the braid arrangement, whose regions are in bijective correspondence with the permutations of $\{1,2, \ldots, n\}$, their theory specializes precisely to the "self-organizing", or "dynamic", one-shelf library that we just described. The theory was later further generalized by Brown [13, 14] to a class of semigroups.

The genesis of this paper is the question: what about random walks on complex hyperplane arrangements? It is of course not at all clear what is meant. The complement in $\mathbb{C}^{d}$ of the union of a finite collection of hyperplanes is a $2 d$ dimensional manifold, so what determines a finite Markov chain?

The idea is to consider not the complement itself, but rather a certain finite cell complex determining the complement up to homotopy type. In addition, we need that this complex extends to a cell complex for the whole singularity link, since much of the probability mass is typically placed in that extension. Such complexes were introduced by Ziegler and the author in [11. The construction and basic properties partly run parallel to a similar construction in the real case, well-known from the theory of oriented matroids. 
The complex hyperplane walks take place on such cell complexes in a manner that will be described in Section 4.3. These cell complexes have a semigroup structure to which the theory of Brown [13] applies. Thus we get results for complex hyperplane walks analogous to those for the real case.

As mentioned, when specialized to the real braid arrangement the general theory of walks on real arrangements leads to the one-shelf dynamic library. What happens when we similarly specialize random walks on complex arrangements to the complex braid arrangement? The pleasant answer is that we are led to Markov chains modelling dynamic libraries with several shelves. These are selforganizing libraries where the books are placed on different shelves according to some classification (combinatorics books, geometry books, etc.), and not only the books on each shelf but also the shelves themselves are permuted in the steps of the Markov chain. Depending on the distribution of probability mass there are different versions.

Here is one. Say that a customer withdraws a subset $E \subseteq[n]$ of books from the library. The books are replaced in the following way. Permute the shelves so that the ones that contain one of the books from $E$ become the top ones, maintaining the induced order among them and among the remaining shelves, which are now at the bottom. Then, on each shelf move the books from $E$ to the beginning of the shelf, where they are placed in the induced order.

The exact description is given in Section 4.4. These Markov chains may be of interest also for file management applications in computer science.

In this paper we take a somewhat leisurely walk through the territory leading to complex hyperplane walks, recalling and assembling results along the way that in the end lead to the desired conclusions. We are not seeking the greatest generality, the aim is rather for simplicity of statements and illuminating ideas through special cases. Some proofs that would interfere with this aspiration are banished to an appendix.

Several topics touched upon in this paper relate to joint work with László Lovász. This is the case for the $k$-equal arrangements [8] in Section 2.4 and for the greedoids [6] in Section 4.5. It is a pleasure to thank Laci for all the pleasant collaborations and interesting discussions over many years.

Also, I am grateful to Persi Diaconis for inspiration and encouragement, and to Jakob Jonsson for helpful remarks.

\section{REAL HYPERPLANE ARRANGEMENTS}

We review the basic facts about real hyperplane arrangements. This material is described in greater detail in many places, for instance in [7] and [19], to where we refer for more detailed information. Also, we adhere to the notation for posets and lattices in [20].

2.1. Basics. Let $\ell_{1}, \ldots, \ell_{t}$ be linear forms on $\mathbb{R}^{d}$, and $H_{i}=\left\{x: \ell_{i}(x)=0\right\} \subseteq \mathbb{R}^{d}$ the corresponding hyperplanes. We call $\mathcal{A}=\left\{H_{1}, \ldots, H_{t}\right\}$ a real hyperplane arrangement. The arrangement is essential if $\cap H_{i}=\{0\}$, and we usually assume that this is the case. 
The complement $M_{\mathcal{A}}=\mathbb{R}^{d} \backslash \cup \mathcal{A}$ consists of a collection $C_{\mathcal{A}}$ of open convex cones $R_{i}$ called regions. They are the connected components of the decomposition $M_{\mathcal{A}}=\biguplus R_{i}$ into contractible pieces.

With $\mathcal{A}$ we associate its intersection lattice $L_{\mathcal{A}}$, consisting of all intersections of subfamilies of hyperplanes $H_{i}$ ordered by set inclusion. Each subspace belonging to $L_{\mathcal{A}}$ can be represented by the set of hyperplanes from $\mathcal{A}$ whose intersection it is. In this way the elements of $L_{\mathcal{A}}$ can be viewed either as subsets of $\mathbb{R}^{d}$ or as subsets of $\mathcal{A}$. The latter is for simplicity encoded as subsets of $[n]$ via the labeling $i \leftrightarrow H_{i}$.

Let $L_{\mathcal{A}}^{\mathrm{op}}$ denote $L_{\mathcal{A}}$ with the opposite partial order, so in $L_{\mathcal{A}}^{\mathrm{op}}$ the subspaces of $\mathbb{R}^{d}$ are ordered by reverse inclusion. This is a geometric lattice, whose atoms are the hyperplanes $H_{i}$.

The number of regions of $\mathcal{A}$ is determined by $L_{\mathcal{A}}$ via its Möbius function in the following way.

Theorem 2.1 (Zaslavsky [22]). $\left|C_{\mathcal{A}}\right|=\sum_{x \in L_{\mathcal{A}}}|\mu(x, \widehat{1})|$

There is a useful way to encode the position of a point $x \in \mathbb{R}^{d}$ with respect to $\mathcal{A}$. Define the sign vector (position vector) $\sigma(x)=\left\{\sigma_{1}, \ldots, \sigma_{t}\right\} \in\{0,+,-\}^{t}$ by

$$
\sigma_{i} \stackrel{\text { def }}{=} \begin{cases}0, & \text { if } \ell_{i}(x)=0 \\ +, & \text { if } \ell_{i}(x)>0 \\ -, & \text { if } \ell_{i}(x)<0\end{cases}
$$

In words, the $i$ th entry $\sigma_{i}$ of the sign vector $\sigma(x)$ tells us whether the point $x$ is on the hyperplane $H_{i}$, or on its positive resp. negative side.

Let $F_{\mathcal{A}} \stackrel{\text { def }}{=} \sigma\left(\mathbb{R}^{d}\right) \subseteq\{+,-, 0\}^{t}$ and make this collection of sign vectors into a poset by componentwise ordering via

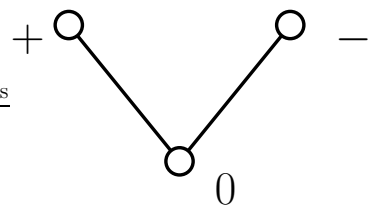

Thus, we have a surjective map $\sigma: \mathbb{R}^{d} \rightarrow F_{\mathcal{A}}$. Note that $F_{\mathcal{A}}$, called the face semilattice, has minimum element $(0, \ldots, 0)$ and its maximal elements $F_{\mathcal{A}} \cap$ $\{+,-\}^{t}$ are in bijective correspondence with the regions, as is illustrated in Figure 1 .
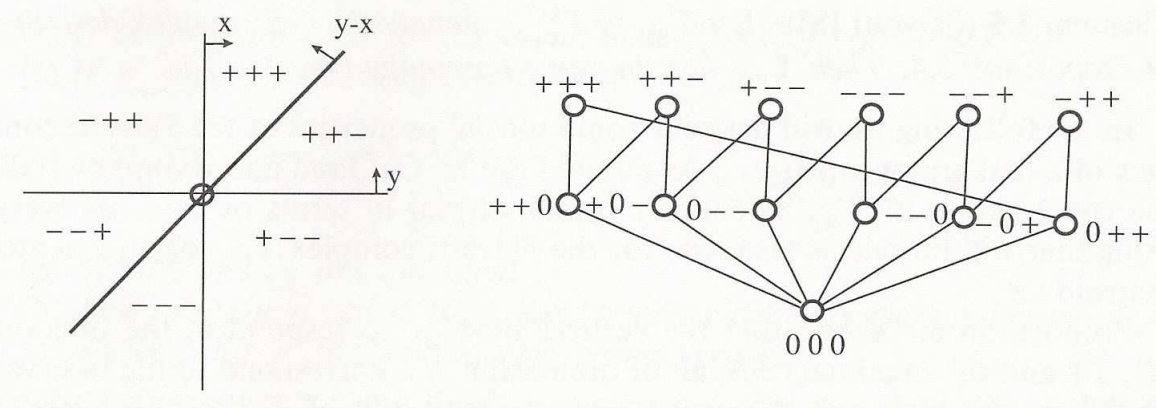
Figure 1. Face semilattice of an arrangement of three lines in $\mathbb{R}^{2}$.

The composition $X \circ Y$ of two sign vectors $X, Y \in\{0,+,-\}^{t}$ is defined by

$$
(X \circ Y)_{i} \stackrel{\text { def }}{=}\left\{\begin{array}{l}
X_{i}, \text { if } X_{i} \neq 0 \\
Y_{i}, \text { if } X_{i}=0
\end{array}\right.
$$

This operation on $\{0,+,-\}^{t}$ is associative, idempotent, and has unit element $(0, \ldots, 0)$. Furthermore, $F_{\mathcal{A}}$ forms a closed subsystem: if $X, Y \in F_{\mathcal{A}}$ then $X \circ Y \in$ $F_{\mathcal{A}}$. Here is the geometric reason: choose points $x, y \in \mathbb{R}^{d}$ such that $\sigma(x)=X$ and $\sigma(y)=Y$. Move a small distance from $x$ along the straight line segment from $x$ to $y$. The point $z$ reached has the position $\sigma(z)=X \circ Y$.

Hence,

$$
\left(F_{\mathcal{A}}, \circ\right) \text { is an idempotent semigroup. }
$$

The combinatorics of sign vectors is systematically developed in oriented matroid theory, where the elements of $F_{\mathcal{A}}$ are called "covectors" and the system $\left(F_{\mathcal{A}}, \circ\right)$ is the basis for one of the fundamental axiom systems, see [7, Section 3.7].

There is an important span map

$$
\text { span : } F_{\mathcal{A}} \rightarrow L_{\mathcal{A}}
$$

which can be characterized in two ways. Combinatorially, it sends the signvector $X$ to the set of positions of its non-zero components (a subset of $[n]$ ). Geometrically, it sends the cone $\sigma^{-1}(X)$ to its linear span.

The span map is a rank-preserving and order-preserving semigroup map, meaning that

$$
\begin{aligned}
\operatorname{rk}_{F_{\mathcal{A}}}(X) & =\operatorname{rk}_{L_{\mathcal{A}}}(\operatorname{span}(X)) \\
X \leq Y & \Rightarrow \operatorname{span}(X) \leq \operatorname{span}(Y) \\
\operatorname{span}(X \circ Y) & =\operatorname{span}(X) \vee \operatorname{span}(Y)
\end{aligned}
$$

Also, we have that

$$
\begin{aligned}
& X \circ Y=Y \quad \Leftrightarrow \quad X \leq Y \\
& X \circ Y=X \quad \Leftrightarrow \quad \operatorname{span}(Y) \leq \operatorname{span}(X)
\end{aligned}
$$

2.2. The braid arrangement. The braid arrangement $\mathcal{B}_{n}=\left\{x_{i}-x_{j} \mid 1 \leq i<\right.$ $j \leq n\}$ in $\mathbb{R}^{n}$ plays an important role in this paper, due to its close connections with the combinatorics of permutations and partitions. The hyperplanes in $\mathcal{B}_{n}$ all contain the diagonal line $(t, t, \ldots, t)$. By intersecting with the hyperplane orthogonal to this line we get an essential arrangement, now in $\mathbb{R}^{d-1}$.

The intersection lattice $L_{\mathcal{B}_{n}}$ is isomorphic to the partition lattice $\Pi_{n}$, i.e. the partitions of the set $[n]$ ordered by reverse refinement. The correspondence between a set partition and a subspace obtained by intersecting some hyperplanes 
$x_{i}-x_{j}$ is easily understood from examples:

$$
(134|27| 5 \mid 6) \leftrightarrow\left\{\begin{array}{l}
x_{1}=x_{3}=x_{4} \\
x_{2}=x_{7}
\end{array}\right.
$$

and

$$
(1345 \mid 267)<(134|27| 5 \mid 6) \text {. }
$$

The face semilattice $F_{\mathcal{B}_{n}}$ is isomorphic to the meet-semilattice of ordered set partitions $\Pi_{n}^{\text {ord }}$ (so, the order of the blocks matters), ordered by reverse refinement. For instance,

$$
\langle 134|6| 27 \mid 5\rangle \leftrightarrow\left\{\begin{array}{l}
x_{1}=x_{3}=x_{4} \\
x_{4}<x_{6}<x_{2} \\
x_{2}=x_{7} \\
x_{7}<x_{5}
\end{array}\right.
$$

and

$$
\langle 1346 \mid 257\rangle<\langle 134|6| 27 \mid 5\rangle \text {. }
$$

Under this correspondence the regions of $\mathbb{R}^{n-1} \backslash \cup \mathcal{B}_{n}$ are in bijection with the ordered partitions into singleton sets, or in other words, with the permutations of the set $[n]$. The span map (2.2) is the map $\Pi_{n}^{\text {ord }} \rightarrow \Pi_{n}$ that sends an ordered partition $\langle\ldots\rangle$ to an unordered partition $(.$.$) by forgetting the ordering of its$ blocks.

Composition in $F_{\mathcal{B}_{n}}$ has the following description. If $X=\left\langle X_{1}, \ldots, X_{p}\right\rangle$ and $Y=\left\langle Y_{1}, \ldots, Y_{q}\right\rangle$ are ordered partitions of $[n]$, then $X \circ Y=\left\langle X_{i} \cap Y_{j}\right\rangle$ with the blocks ordered by the lexicographic order of the pairs of indices $(i, j)$. For instance,

$$
\langle 257|3| 146\rangle \circ\langle 17|25| 346\rangle=\langle 7|25| 3|1| 46\rangle,
$$

as can conveniently be seen from the computation table

\begin{tabular}{c||c|c|c||}
$\circ$ & 1,7 & 2,5 & $3,4,6$ \\
\hline \hline $2,5,7$ & 7 & 2,5 & \\
\hline 3 & & & 3 \\
\hline $1,4,6$ & 1 & & 4,6 \\
\hline \hline
\end{tabular}

2.3. Cell complexes and zonotopes. The whole idea of random walks on complex hyperplane arrangements rests on the idea of walking on the cells of an associated cell complex. We therefore review the construction used in [11] of such cell complexes. The basic idea is given together with two applications. The first one is the construction of cell complexes for the complement of a linear subspace arrangement in $\mathbb{R}^{d}$ at the end of this section. The other is the construction of cell complexes for hyperplane arrangements in $\mathbb{C}^{d}$, to which we return in Section 3.2. See e.g. [4] for topological terminology.

A regular cell decomposition $\Gamma$ of the unit sphere $S^{d-1}$ is said to be $P L$ if its barycentric subdivision (equivalently, the order complex of its face poset) is a 
piecewise linear triangulation of $S^{d-1}$. Here is a simple combinatorial procedure for producing regular cell complexes of certain specific homotopy types from posets.

Proposition 2.2. [11, Prop. 3.1] Suppose that $\Gamma$ is a PL regular cell decomposition of $S^{d-1}$, with face poset $F_{\Gamma}$. Let $T \subseteq S^{d-1}$ be a subspace of the sphere such that $T=\cup_{\tau \in G} \tau$ for some order ideal $G \subseteq F_{\Gamma}$. Then the poset $\left(F_{\Gamma} \backslash G\right)^{\text {op }}$ is the face poset of a regular cell complex having the homotopy type of the complement $S^{d-1} \backslash T$.

Now, let $\mathcal{A}$ be an essential hyperplane arrangement in $\mathbb{R}^{d}$. For a general sign vector $X \in F_{\mathcal{A}}$ the set $\sigma^{-1}(X)$ is a convex cone in $\mathbb{R}^{d}$ which is open in its linear span. Let $\tau_{X} \stackrel{\text { def }}{=} \sigma^{-1}(X) \cap S^{d-1}$. The sets $\tau_{X}$, for $X \in F_{\mathcal{A}} \backslash \widehat{0}$, partition the the unit sphere and are in fact the open cells of a regular CW decomposition of $S^{d-1}$. Furthermore, the inclusion relation of their closures $\overline{\tau_{X}}$ coincides with the partial order we have defined on $F_{\mathcal{A}}$. Thus, $F_{\mathcal{A}} \backslash \widehat{0}$ is the face poset of a regular cell decomposition $\Gamma_{\mathcal{A}}$ of the unit sphere in $\mathbb{R}^{d}$, namely the cell decomposition naturally cut out by the hyperplanes.

The cell complex $\Gamma_{\mathcal{A}}=\left\{\tau_{X}\right\}_{X \in F_{\mathcal{A}}}$ induced by a hyperplane arrangement $\mathcal{A}$ is PL. Thus, via Proposition 2.2 we can construct cell complexes determining the complement of a subcomplex up to homotopy type. Combinatorially the description is simple: erase from the face poset $F_{\mathcal{A}}$ all the cells that belong to the given subcomplex and then turn the remaining subposet upside down. Done!

The cell complexes constructed this way from a hyperplane arrangement $\mathcal{A}$ can be geometrically realized on the boundary of an associated convex polytope. Namely, with $\mathcal{A}$ is associated its zonotope $\mathbf{Z}_{\mathcal{A}}=\left[-e_{1}, e_{1}\right] \oplus \cdots \oplus\left[-e_{t}, e_{t}\right]$. Here $e_{i}$ is a normal vector in $\mathbb{R}^{d}$ to the hyperplane $H_{i}$ and the right-hand side denotes Minkowski sum of centrally symmetric line segments. Thus, $\mathbf{Z}_{\mathcal{A}}$ is a centrally symmetric convex polytope, determined this way up to combinatorial equivalence. A key property of $\mathbf{Z}_{\mathcal{A}}$ is that there exists an order-reversing bijection between the faces on its boundary and the cells of $\Gamma_{\mathcal{A}}$. In other words, the poset of proper faces of $\mathbf{Z}_{\mathcal{A}}$ is isomorphic to the opposite of the face poset of $\mathcal{A}$ :

$$
F_{\mathbf{z}_{\mathcal{A}}} \cong\left(F_{\mathcal{A}} \backslash \widehat{0}\right)^{\mathrm{op}}
$$

Suppose that $\mathcal{A}$ is an arrangement of linear subspaces of arbitrary dimensions in $\mathbb{R}^{d}$. Say that we want to construct a cell complex having the homotopy type of its complement $\mathbb{R}^{d} \backslash \cup \mathcal{A}$. This complement is by radial projection homotopy equivalent to its intersection with the unit sphere $S^{d-1}$. Therefore the preceding construction is applicable. We just have to choose an auxiliary hyperplane arrangement $\mathcal{H}$ into which $\mathcal{A}$ embeds, meaning that each subspace in $\mathcal{A}$ is the intersection of some of the hyperplanes from $\mathcal{H}$. This is clearly always possible. Putting the various pieces of information together and applying Proposition 2.2 we obtain the following description.

Theorem 2.3. 11] Let $\mathcal{A}$ be an arrangement of linear subspaces in $\mathbb{R}^{d}$. Choose a hyperplane arrangement $\mathcal{H}$ into which $\mathcal{A}$ embeds. Then the complement $\mathbb{R}^{d} \backslash \cup \mathcal{A}$ 
has the homotopy type of a subcomplex $\mathbf{Z}_{\mathcal{H}, \mathcal{A}}$ of the boundary of the zonotope $\mathbf{Z}_{\mathcal{H}}$. The complex $\mathbf{Z}_{\mathcal{H}, \mathcal{A}}$ is obtained by deleting from the boundary of $\mathbf{Z}_{\mathcal{H}}$ all faces that correspond to cells $\tau_{X}$ contained in $\cup \mathcal{A}$.

2.4. The permutohedron and the $k$-equal arrangements. We illustrate the general constructions of the preceding section by applying them to the $k$-equal arrangements $\mathcal{A}_{n, k}=\left\{x_{i_{1}}=x_{i_{2}}=\cdots=x_{i_{k}}: 1 \leq i_{1}<i_{2}<\cdots<i_{k} \leq n\right\}$ in $\mathbb{R}^{n}$. The topology of their complements play a crucial role in the solution of a complexity-theoretic problem in joint work with Lovász and Yao [8, 9]. See also [10], where their homology groups were computed. The $k$-equal arrangements embed into the braid arrangement (the $k=2$ case), so Theorem 2.3 is applicable. It tells us that, up to homotopy type, the topology of the complement of the $k$ equal arrangement $\mathcal{A}_{n, k}$ is realized by some subcomplex of the zonotope of the braid arrangement. This subcomplex can be very explicitly described.

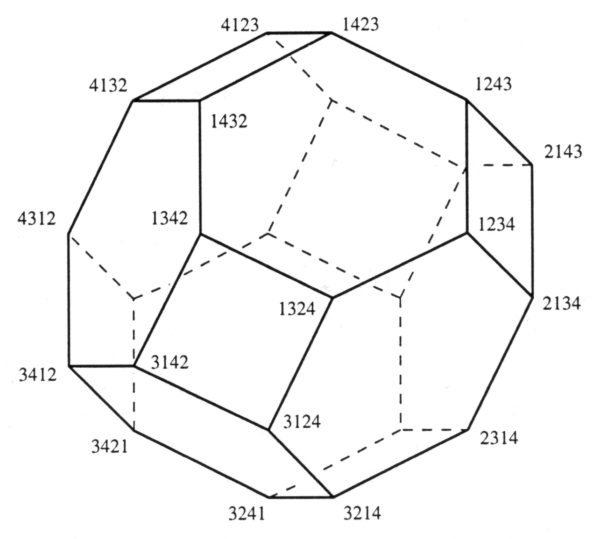

Figure 2. The permutohedron $\mathbf{Z}_{4}^{\text {perm }}$.

The zonotope of the braid arrangement $\mathcal{B}_{n}$ is the permutohedron $\mathbf{Z}_{n}^{\text {perm }}$, that is, the convex hull of the $n$ ! points in $\mathbb{R}^{n}$ whose coordinates are given by a permutation of the numbers $1,2, \ldots, n$. Its $n$ ! vertices are in bijection with the $n$ ! regions of $\mathcal{B}_{n}$, in accordance with the duality (2.9)).

We want to describe the subcomplex $\mathbf{Z}_{\mathcal{A}_{n, k}}$ of the boundary of $\mathbf{Z}_{n}^{\text {perm }}$ which is homotopy equivalent to the complement $\mathcal{M}_{n, k}$ of $\mathcal{A}_{n, k}$.

For this one argues as follows, keeping Section 2.2 in fresh memory. Let $f$ : $\Pi_{n}^{\text {ord }} \rightarrow \Pi_{n}$ be the span map, i.e., the forgetful map that sends an ordered partition of $[n]$ to the corresponding unordered partition. The set $\Pi_{n}^{\text {ord }} \backslash \widehat{0}$ ordered by refinement is the poset of proper faces of the permutohedron $\mathbf{Z}_{n}^{\text {perm }}$, whereas the set $\Pi_{n}$ ordered by refinement is the opposite of intersection lattice of the braid arrangement. The image $f(\pi)$ for $\pi \in \Pi_{n}^{\text {ord }}$ is a partition determining the span of the corresponding cell (i.e., the smallest intersection subspace of the braid arrangement in which the cell is contained). More precisely, the span of $\pi$ is the subspace obtained by setting $x_{i_{1}}=x_{i_{2}}=\cdots=x_{i_{j}}$ for each block $\left\{i_{1}, i_{2} \ldots, i_{j}\right\}$ 
of $\pi$. Thus, a cell $\pi \in \Pi_{n}^{\text {ord }}$ lies in the union of the $k$-equal arrangement if and only if some block has size at least $k$.

It follows that the complex $\mathbf{Z}_{\mathcal{B}_{n}, \mathcal{A}_{n, k}}$ consists of those cells on the boundary of the permutohedron $\mathbf{Z}_{n}^{\text {perm }}$ that correspond to ordered partitions with all blocks of size less than $k$. If an ordered partition has blocks of sizes $b_{1}, \ldots, b_{e}$, then the corresponding face of $\mathbf{Z}_{n}^{\text {perm }}$ is the product of smaller permutohedra of dimensions $b_{1}-1, \ldots, b_{e}-1$. Therefore, the final description of the cell complex $\mathbf{Z}_{\mathcal{B}_{n} \mathcal{A}_{n, k}}$ is that one should delete from $\mathbf{Z}_{n}^{\text {perm }}$ all faces that contain a $q$-dimensional permutohedron, for $q \geq k-1$, in its decomposition.

We are led to the following result, obtained independently by E. Babson for $k=3$ (see [1]) and the author [5].

Theorem 2.4. Delete from the boundary of the permutohedron $\mathbf{Z}_{n}^{\text {perm }}$ every face that contains a d-dimensional permutohedron, $d \geq k-1$, in its decomposition. Then the remaining subcomplex has the homotopy type of the complement of the $k$-equal arrangement.

Thus, for $k=2$ one deletes everything but the vertices, for $k=3$ one deletes all cells except those that are products of edges (equivalently, keep only the cubical faces), for $k=4$ one deletes all cells except those that are products of edges (1-dimensional zonotope) and hexagons (2-dimensional zonotope), and so on.

The case $k=3$ is especially interesting. The complex is in that case cubical. In particular, the fundamental group of $\mathcal{M}_{n, 3}$ is the same as the fundamental group of the cell complex obtained from the graph (1-skeleton) of $\mathbf{Z}_{n}^{\text {perm }}$ by gluing a 2-cell (membrane) into every 4-cycle.

Remark 2.5. What was just said is part of a more general result about gluing 2-cells into 4-cycles of a zonotopal graph.

Let $\mathcal{H}$ be an arbitrary central and essential hyperplane arrangement, and let $\mathcal{A}$ be the subspace arrangement consisting of codimension 2 intersections of 3 or more planes from $\mathcal{H}$ (assuming that there are such).

Next, let $G$ be the 1-skeleton of the zonotope $\mathbf{Z}_{\mathcal{H}}$. The 2-cells of $\mathbf{Z}_{\mathcal{H}}$ are $2 m$-gons (corresponding to codimension 2 subspaces where $m$ planes meet). Let $\Gamma_{\mathcal{A}}$ be the cell complex obtained by gluing 2-cells into the 4-cycles of the graph $G$. Then the general construction above shows (since fundamental groups live on 2-skeleta) that the fundamental group of $\Gamma_{\mathcal{A}}$ is isomorphic to that of the complement $\mathcal{M}_{\mathcal{A}}$.

One can go on and describe the higher-dimensional cells needed to obtain a cell complex having the homotopy type of the complement of such a codimension 2 arrangement $\mathcal{A}$. They are all the cubes in the boundary of $\mathbf{Z}_{\mathcal{H}}$, just like for the special case of the 3 -equal arrangement.

Remark 2.6. The two-dimensional faces of $\mathbf{Z}_{n}^{\text {perm }}$ are either 4-gons or 6-gons. What happens if we take the graph of the permutohedron and glue in only the hexagonal 2-cells? The answer is that we get a two-dimensional cell complex whose fundamental group is isomorphic to that of the complement of another subspace arrangement, namely the arrangement $\mathcal{A}_{[2,2]}$ consisting of codimension 
2 subspaces of $\mathbb{R}^{n}$ obtained as intersections of pairs of hyperplanes $x_{i}=x_{j}$ and $x_{k}=x_{l}$, for all distinct $i, j, k, l$. Actually, for $\mathcal{A}_{[2,2]}$ a stronger statement is true: the 2-dimensional cell complex described (i.e. the permutohedron graph plus all hexagonal 2-cells) has the homotopy type of its complement.

It is an interesting fact that the codimension 2 arrangements $\mathcal{A}_{n, 3}$ and $\mathcal{A}_{[2,2]}$, corresponding to the two ways of gluing 2-cells into the permutohedron graph, share a significant topological property, namely that their complements are $\mathrm{K}(\pi, 1)$ spaces. See Khovanov [17].

\section{Complex hyperplane ARRANGEMENTS}

We now move the discussion to complex space. To begin with many of the concepts and results are parallel to the real case. But new interesting features soon start to appear. This whole chapter summarizes material from [11].

3.1. Basics. We call $\mathcal{A}=\left\{H_{1}, \ldots, H_{t}\right\}$ a complex hyperplane arrangement if $H_{i}=\left\{z: \ell_{i}(z)=0\right\} \subseteq \mathbb{C}^{d}$ for some linear forms $\ell_{1}, \ldots, \ell_{t}$ on $\mathbb{C}^{d}$. A particular choice of defining linear forms is assumed throughout, so we can also write $\mathcal{A}=$ $\left\{\ell_{1}, \ldots, \ell_{t}\right\}$. The arrangement is essential if $\cap H_{i}=\{0\}$, and we usually assume that this is the case. The real and imaginary parts of $w=x+i y \in \mathbb{C}$ are denoted, respectively, by $\Re(w)=x$ and $\Im(w)=y$.

The position of a point $z \in \mathbb{C}^{d}$ with respect to $\mathcal{A}$ is combinatorially encoded in the following way. Define the sign vector (position vector) $\sigma(z)=\left\{\sigma_{1}, \ldots, \sigma_{t}\right\} \in$ $\{0,+,-, i, j\}^{t}$ by

$$
\sigma_{i}= \begin{cases}0, & \text { if } \ell_{i}(z)=0 \\ +, & \text { if } \Im\left(\ell_{i}(z)\right)=0, \Re\left(\ell_{i}(x)>0\right. \\ -, & \text { if } \Im\left(\ell_{i}(z)\right)=0, \Re\left(\ell_{i}(x)<0\right. \\ i, & \text { if } \Im\left(\ell_{i}(z)\right)>0 \\ j, & \text { if } \Im\left(\ell_{i}(z)\right)<0\end{cases}
$$

Let $F_{\mathcal{A}} \stackrel{\text { def }}{=} \sigma\left(\mathbb{C}^{d}\right) \subseteq\{0,+,-, i, j\}^{t}$ and make this collection of sign vectors into a poset, called the face poset, by componentwise ordering via

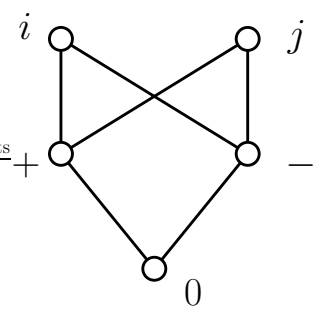

Proposition 3.1. [11]

(1) $F_{\mathcal{A}}$ is a ranked poset of length $2 d$. Its unique minimal element is 0.

(2) The maximal elements of $F_{\mathcal{A}}$ are the sign vectors in $F_{\mathcal{A}} \cap\{i, j\}^{t}$.

(3) $\mu(Z, W)=(-1)^{\mathrm{rk}(W)-\mathrm{rk}(Z)}$, for all $Z \leq W$ in $F_{\mathcal{A}} \cup \widehat{1}$. 
Figure 3 (borrowed from [11]), shows the face poset of $\mathcal{A}=\{z, w, w-z\}$ in $\mathbb{C}^{2}$. The reason for marking the elements not containing any zero with filled dots becomes clear in Section 3.2

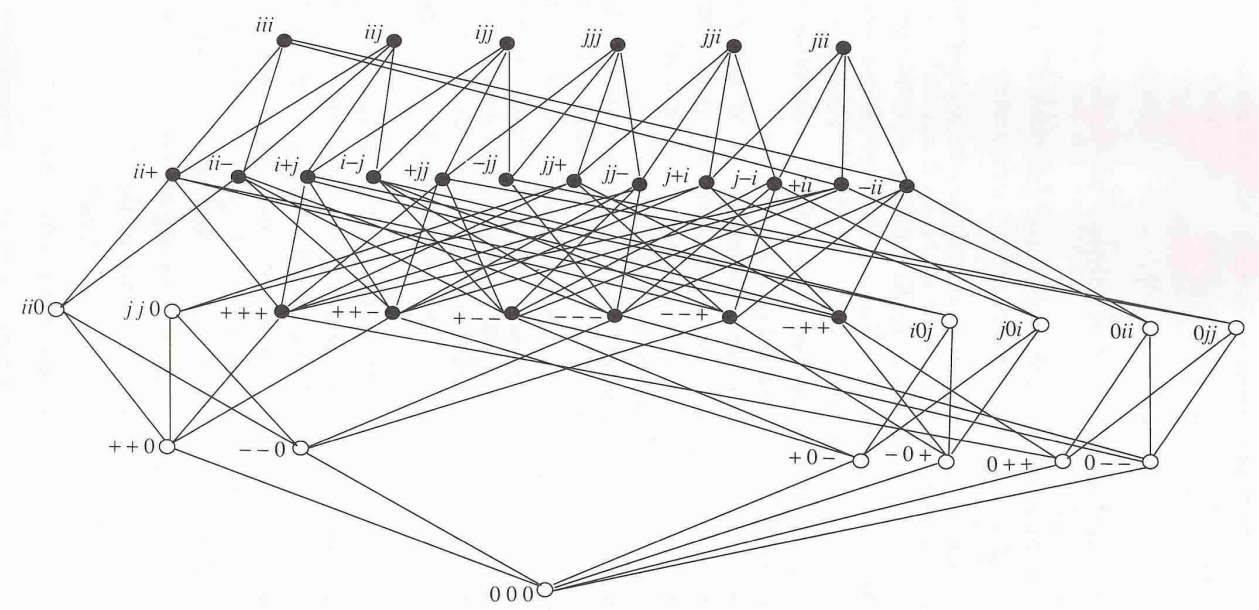

Figure 3. Face poset of an arrangement of three lines in $\mathbb{C}^{2}$.

The composition of two complex sign vectors $Z \circ W \in\{0,+,-, i, j\}^{t}$ is defined by

$$
(Z \circ W)_{i}= \begin{cases}Z_{i}, & \text { if } W_{i} \ngtr Z_{i} \\ W_{i}, & \text { if } W_{i}>Z_{i}\end{cases}
$$

Just as in the corresponding real case this operation on $\{0,+,-, i, j\}^{t}$ is associative, idempotent, and has unit element $(0, \ldots, 0)$. Also, for geometric reasons (analogous to the ones in the real case) $X, Y \in F_{\mathcal{A}}$ implies that $X \circ Y \in F_{\mathcal{A}}$ Hence,

$$
\left(F_{\mathcal{A}}, \circ\right) \text { is an idempotent semigroup. }
$$

For complex arrangements the notion of intersection lattice splits into two.

1. The intersection lattice $L_{\mathcal{A}}$ consists of all intersections of subfamilies of hyperplanes $H_{i}$ ordered by set inclusion.

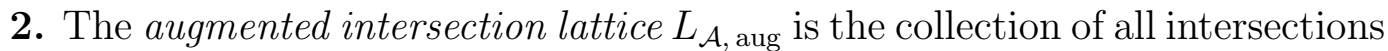
of subfamilies of the augmented arrangement

$$
\mathcal{A}_{\text {aug }}=\left\{H_{1}, \ldots, H_{t}, H_{1}^{\mathbb{R}}, \ldots, H_{t}^{\mathbb{R}}\right\}
$$

ordered by set inclusion. Here, $H_{i}^{\mathbb{R}} \stackrel{\text { def }}{=}\left\{z \in \mathbb{C}^{d}: \Im\left(\ell_{i}(z)\right)=0\right\}$ is a $(2 d-1)$ dimensional real hyperplane in $\mathbb{C}^{d} \cong \mathbb{R}^{2 d}$ containing $H_{i}$.

Again as in the real case, we denote by $L_{\mathcal{A}}^{\mathrm{op}}$ and $L_{\mathcal{A} \text {, aug }}^{\mathrm{op}}$ the opposite lattices, obtained by reversing the partial order.

Proposition 3.2. (1) $L_{\mathcal{A}}^{\mathrm{op}}$ is a geometric lattice of length $d$.

(2) $L_{\mathcal{A} \text {, aug }}^{\mathrm{op}}$ is a semimodular lattice of length $2 d$. 
There is a span map

$$
\operatorname{span}: F_{\mathcal{A}} \rightarrow L_{\mathcal{A} \text {, aug }}
$$

defined by sending the convex cone $\sigma^{-1}(Z)$, for $Z \in F_{\mathcal{A}}$, to the intersection of all subspaces in $\mathcal{A}_{\text {aug }}$ that contain $\sigma^{-1}(Z)$.

This map preserves poset and semigroup structure as well as poset rank.

Proposition 3.3. 11]

$$
\begin{aligned}
\operatorname{rk}_{F_{\mathcal{A}}}(Z) & =\operatorname{rk}_{L_{\mathcal{A}, \text { aug }}}(\operatorname{span}(Z)) \\
Z \leq W & \Rightarrow W \circ Z=W \Leftrightarrow \operatorname{span}(Z) \leq \operatorname{span}(W) \\
\operatorname{span}(Z \circ W) & =\operatorname{span}(Z) \vee \operatorname{span}(W)
\end{aligned}
$$

3.2. Cell complexes. The complement $M_{\mathcal{A}}=\mathbb{C}^{d} \backslash \cup \mathcal{A}$ is a complex manifold of real dimension $2 d$. There is a huge literature on the topology of such spaces, see e.g. [19]. Among the basic results we mention that the Betti numbers of $M_{\mathcal{A}}$ are determined by $L_{\mathcal{A}}$ via its Möbius function in the following way.

Theorem 3.4. [19, p. 20] $\beta_{i}\left(M_{\mathcal{A}}\right)=\sum_{x \in L_{\mathcal{A}}: \operatorname{rk}(x)=d-i}|\mu(x, \widehat{1})|$

Let $\mathcal{A}$ be an essential complex hyperplane arrangement in $\mathbb{C}^{d}$, as before. For every sign vector $Z \in F_{\mathcal{A}} \backslash 0$ the inverse image $\sigma^{-1}(Z)$ is a relative-open convex cone in $\mathbb{C}^{d}$. The intersections of these cones with the unit sphere $S^{2 d-1}$ in $\mathbb{C}^{d}$ are the open cells of a PL regular cell decomposition of $S^{2 d-1}$ whose face poset is isomorphic to $F_{\mathcal{A}}$. Hence, as an application of Proposition 2.2 we get part (3) of the following result. Part (2) can be seen from the fact that $x$ is an $\operatorname{rk}(x)$ dimensional linear subspace, so $x \cap S^{2 d-1}$ is an $(\operatorname{rk}(x)-1)$-dimensional sphere, for all $x \in L_{\mathcal{A} \text {, aug }} \backslash \widehat{0}$, where "rk" denotes poset rank in $L_{\mathcal{A} \text {, aug. }}$.

Theorem 3.5. 11]

(1) The poset $F_{\mathcal{A}}$ is the face poset of a regular cell decomposition of the unit sphere in $\mathbb{R}^{2 d} \cong \mathbb{C}^{d}$.

(2) The subposet $\operatorname{span}^{-1}\left(\left(L_{\mathcal{A}, \text { aug }}\right)_{\leq x}\right)$ is the face poset of a regular cell decomposition of the sphere $S^{\mathrm{rk}(x)-1}$, for all $x \in L_{\mathcal{A} \text {, aug }} \backslash \widehat{0}$.

(3) The subposet $C_{\mathcal{A}} \stackrel{\text { def }}{=} F_{\mathcal{A}} \cap\{+,-, i, j\}^{t}$, with opposite order, is the face poset of a regular cell complex having the homotopy type of the complement $M_{\mathcal{A}}$.

For an example, have a look at Figure 3. The sign vectors in $F_{\mathcal{A}}$ that lack a zero component are shown by filled dots. Hence, the cell complex $C_{\mathcal{A}}$ can be viewed by turning the page upside-down and looking at the subposet of filled dots only.

Combining some of this topological information with Theorem 5.1 of the Appendix we obtain the following analogue of Zaslavsky's theorem 2.1 for the number of maximal cells in the complex case.

Theorem 3.6. $\left|\max \left(F_{\mathcal{A}}\right)\right|=\sum_{x \in L_{\mathcal{A} \text {, aug }}}|\mu(x, \widehat{1})|$ 


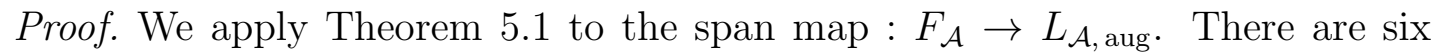
conditions to verify. With the exception of (5), they all follows from Propositions 3.1 and 3.2 . Condition (5) is the consequence for the Euler characteristic of Theorem 3.5(2).

3.3. Complexified $\mathbb{R}$-arrangements. This section concerns the special case when all the linear forms $\ell_{i}(z)$ have real coefficients. The forms then define both a real arrangement $\mathcal{A}^{\mathbb{R}}$ in $\mathbb{R}^{d}$ and a complex arrangement $\mathcal{A}^{\mathbb{C}}$ in $\mathbb{C}^{d}$. These are of course related, and we here summarize what expression this relation takes for the combinatorial structures of interest.

First a few observations about complex sign vectors. A sign vector $Z$ is called real if all its entries come from $\{0,+,-\}$. Every complex sign vector $Z$ can be obtained as a composition $Z=X \circ i Y$ for two real sign vectors $X$ and $Y$. Only the vector $Y$ is unique in this decomposition.

For any poset $P$, let $\operatorname{Int}(P)$ denote the set of its closed intervals. In the case of the face poset $F_{\mathcal{A}^{\mathbb{R}}}$ of a real arrangement $\mathcal{A}^{\mathbb{R}}$ we make $\operatorname{Int}\left(F_{\mathcal{A}^{\mathbb{R}}}\right)$ into a poset by introducing the following partial order:

$$
[Y, X] \leq[R, S] \leftrightarrow\left\{\begin{array}{l}
Y \leq R \\
R \circ X \leq S
\end{array}\right.
$$

Proposition 3.7. [1] The map $\phi: \operatorname{Int}\left(F_{\mathcal{A}^{\mathbb{R}}}\right) \rightarrow F_{\mathcal{A}^{\mathbb{C}}}$ given by $[Y, X] \mapsto X \circ i Y$ is a poset isomorphism.

For example,

$$
\phi:[(0-+00-),(--+0+-)] \mapsto(-j i 0+j)
$$

Hence, the entire structure of the complex face poset $F_{\mathcal{A}^{\mathbb{C}}}$ can be dealt with in terms of intervals in the real face poset $F_{\mathcal{A}^{\mathbb{R}}}$. In particular, the cells in the complement of $\mathcal{A}$, being the sign vectors without any zero coordinate, get this description.

$$
C_{\mathcal{A}^{\mathbb{C}}} \stackrel{\phi}{\leftrightarrow} \text { intervals }[Y, X] \text { with } X \in \max \left(F_{\mathcal{A}^{\mathbb{R}}}\right)
$$

Composition of complex sign vectors (3.1) takes the following form when translated to intervals:

$$
[Y, X] \circ[R, S]=[Y \circ R, Y \circ R \circ X \circ S]
$$

The augmented intersection lattice $L_{\mathcal{A}^{\mathbb{C}} \text {,aug }}$ is similarly determined by the intervals of $L_{\mathcal{A}^{\mathbb{R}}}$, namely

$$
L_{\mathcal{A}^{\mathbb{C}}, \text { aug }} \cong \operatorname{Int}\left(L_{\mathcal{A}^{\mathbb{R}}}\right),
$$

this time with the partial order defined by

$$
(x, y) \leq\left(x^{\prime}, y^{\prime}\right) \quad \text { if and only if } \quad x \leq x^{\prime} \text { and } y \leq y^{\prime} .
$$

The span map is the natural one

$$
\operatorname{Int}\left(F_{\mathcal{A}^{\mathbb{R}}}\right) \cong F_{\mathcal{A}^{\mathbb{C}}} \rightarrow L_{\mathcal{A}^{\mathbb{C}}, \text { aug }} \cong \operatorname{Int}\left(L_{\mathcal{A}^{\mathbb{R}}}\right)
$$

\footnotetext{
${ }^{1}$ here $i \cdot 0=0, i \cdot+=i, i \cdot-=j$.
} 
sending $[Y, X]$ to $[\operatorname{span}(Y), \operatorname{span}(X)]$. The Möbius function of $\operatorname{Int}\left(L_{\mathcal{A}^{\mathbb{R}}}\right)$ is described in terms of the Möbius function of the lattice $L_{\mathcal{A}^{\mathbb{R}}}$ in Appendix 5.2 .

Example 3.8. The braid arrangement $\mathcal{B}_{n}^{\mathbb{C}}=\left\{x_{i}-x_{j} \mid 1 \leq i<j \leq n\right\}$ in $\mathbb{C}^{n}$ is the complexification of the real braid arrangement, discussed in Section 2.2. Hence we can translate its combinatorics into the language of intervals, as outlined in this section.

We obtain that $\mathcal{B}_{n}^{\mathbb{C}}$ has face semilattice

$$
F_{\mathcal{B}_{n}^{\mathbb{C}}} \cong \operatorname{Int}\left(F_{\mathcal{B}_{n}^{\mathbb{R}}}\right) \cong \operatorname{Int}\left(\Pi_{n}^{\text {ord }}\right)
$$

and augmented intersection lattice

$$
L_{\mathcal{B}_{n}^{\complement}, \text { aug }} \cong \operatorname{Int}\left(L_{\mathcal{B}_{n}^{\mathbb{R}}}\right) \cong \operatorname{Int}\left(\Pi_{n}\right) \text {. }
$$

Thus, the complex sign vectors of $\mathcal{B}_{n}^{\mathbb{C}}$ are encoded into pairs $[Y, X]$ of ordered partitions, where $X$ is an refinement of $Y$. The composition (3.8) is illustrated in this computation table:

\begin{tabular}{c||c|c|c||c|c||c|c||}
$\circ$ & 1 & 3 & 5 & 4 & 7 & 6 & 2 \\
\hline \hline 3,7 & & 3 & & & 7 & & \\
\hline 1 & 1 & & & & & & \\
\hline \hline $2,5,6$ & & & 5 & & & 6 & 2 \\
\hline 4 & & & & 4 & & & \\
\hline \hline
\end{tabular}

from which we read that

$$
\langle 37|1||256| 4\rangle \circ\langle 1|3| 5|| 4|7||6| 2\rangle=\langle 3|1||7||5||4||6| 2\rangle
$$

Here single bars denote the separation of the ground set [7] into ordered blocks according to $X$, and double bars the coarser partition $Y$. The rule is to read off the coarser partition of the composition by ordering the double bar boxes lexicographically, and then read off the refinement by ordering the single bar boxes within each double bar box lexicographically (empty boxes are skipped).

Notice that the cells in the complement of the complex braid arrangement, cf. Theorem 3.5 (3), correspond to block-divided permutations:

$$
\begin{aligned}
C_{\mathcal{B}_{n}^{\complement}} & \leftrightarrow \text { sign vectors } X \circ i Y \text { without zero coordinates } \\
& \leftrightarrow \text { intervals }[Y, X], X \text { maximal } \\
& \leftrightarrow \text { permutations } X \text { divided into ordered blocks } Y
\end{aligned}
$$

\section{RANDOM WALKS}

This chapter begins with a summary of Brown's theory for random walks on a class of semigroups [13]. The motivating example, namely walks on real hyperplane arrangements, is then recalled. After that comes a sequence of applications. 
4.1. Walks on semigroups. A semigroup is a set with an associative composition. We also assume the existence of an identity element, denoted " $e$ ", and we write the composition in multiplicative notation.

Definition 4.1. An LRB semigroup is a finite semigroup $\Sigma$ with identity satisfying

(1) $x^{2}=x$ for all $x \in \Sigma$,

(2) $x y x=x y$ for all $x, y \in \Sigma$.

$A$ left ideal of $\Sigma$ is a subset $I \subseteq \Sigma$ such that $x \in \Sigma, y \in I \Rightarrow x y \in I$.

The acronym LRB stands for "Left-Regular Band", a name by which this class of semigroups is sometimes known in the literature. Brown [13] defined a class of random walks on semigroups of this type. This section summarizes some material from [13], to where we refer for more information, background and references.

Definition 4.2. Let $I$ be a left ideal of $\Sigma$, and let $w$ be a probability distribution on $\Sigma$. A random walk on $I$ is defined in the following way. If the current position of the walk is at an element $y \in I$, then choose $x \in \Sigma$ according to the distribution $w$ and move to $x y$.

Brown's main theorem gives surprisingly exact information about such random walks. In order to be able to state it we need to first introduce two related poset structures.

Let $\Sigma$ be an LRB semigroup. We define a relation " $\leq$ " on $\Sigma$ by

$$
x \leq y \quad \Leftrightarrow \quad x y=y
$$

This turns out to be a partial order relation, so we may think of an LRB semigroup also as a poset. The identity element $e$ is the unique minimal element. The set $\max (\Sigma)$ of maximal elements is a left ideal in $\Sigma$.

There is also another partial order significantly related to $\Sigma$.

Proposition $4.3([13])$. Let $\Sigma$ be an LRB semigroup. Then there exists a unique finite lattice $\Lambda$ and an order-preserving and surjective map

$$
\text { supp : } \Sigma \rightarrow \Lambda
$$

such that for all $x, y \in \Sigma$ :

(1) $\operatorname{supp}(x y)=\operatorname{supp}(x) \vee \operatorname{supp}(y)$

(2) $\operatorname{supp}(x) \leq \operatorname{supp}(y) \Leftrightarrow y x=y$

We call $\Lambda$ the support lattice and supp the support map. Observe that

$$
\operatorname{supp}^{-1}(\widehat{0})=\{e\} \text { and } \operatorname{supp}^{-1}(\widehat{1})=\max (\Sigma)
$$

where $\widehat{0}$ and $\widehat{1}$ denote the bottom and top elements of $\Lambda$. In fact, the following conditions on an element $c \in \Sigma$ are equivalent:

(1) $\operatorname{supp}(c)=\widehat{1}$

(2) $c \in \max (\Sigma)$,

(3) $c x=c$, for all $x \in \Sigma$.

Here is the main result on the random walks of Definition 4.2 . 
Theorem 4.4 (Brown [13]). Let $\Sigma$ be an LRB semigroup and $\Lambda$ its support lattice. Furthermore, let $\left\{w_{x}\right\}$ be a probability distribution on $\Sigma$ and $P_{w}$ the transition matrix of the induced random walk on the ideal $\max (\Sigma)$ :

$$
P_{w}(c, d)=\sum_{x: x c=d} w_{x}
$$

for $c, d \in \max (\Sigma)$. Then,

(1) The matrix $P_{w}$ is diagonalizable.

(2) For each $X \in \Lambda$ there is an eigenvalue $\varepsilon_{X}=\sum_{y: \operatorname{supp}(y) \leq X} w_{y}$.

(3) The multiplicity of the eigenvalue $\varepsilon_{X}$ is $m_{X}=\sum_{Y: Y \geq X} \mu_{\Lambda}(X, Y) c_{Y}$, where $c_{Y} \stackrel{\text { def }}{=}\left|\max \left(\Sigma_{\geq y}\right)\right|$, for any $y \in \operatorname{supp}^{-1}(Y)$.

(4) These are all the eigenvalues of $P$.

(5) Suppose that $\Sigma$ is generated by $\left\{x \in \Sigma: w_{x}>0\right\}$. Then the random walk on $\max (\Sigma)$ has a unique stationary distribution $\pi$.

By Möbius inversion the multiplicities can be determined also from the relations

$$
c_{X}=\sum_{Y: Y \geq X} m_{Y} .
$$

Theorem 4.4 is a generalization from the special case of face semigroups of real hyperplane arangements, to be briefly reviewed in the following section. In that case the theorem emanates from the work of Bidigare, Hanlon and Rockmore [2] and was expanded by Brown and Diaconis [15]. The generalization to LRB semigroups was given by Brown [13, 14].

The cited papers also contain information about the rate of convergence to stationarity and various descriptions of the stationary distribution, e.g. via sampling techniques, see [2, 13, 14, 15, 16] for such information.

The following proposition describes two ways in which smaller LRB semigroups are induced.

Proposition 4.5 ([13]). Let $\Sigma$ be an LRB semigroup with support lattice $\Lambda$. Suppose that $x \in \Sigma$ and $X \in \Lambda$. Then

(1) $\Sigma_{\geq x} \stackrel{\text { def }}{=}\{y \in \Sigma: y \geq x\}$ is an LRB semigroup whose support lattice is the interval $[\operatorname{supp}(x), \widehat{1}]$ in $\Lambda$.

(2) If $\operatorname{supp}(x)=\operatorname{supp}(y)$ then $\Sigma_{\geq x} \cong \Sigma_{\geq y}$.

(3) $\operatorname{Fib}_{\Lambda}(X) \stackrel{\text { def }}{=}\{y \in \Sigma: \operatorname{supp}(y) \leq X\}$ is an LRB semigroup (we call it the fiber semigroup at $X)$, whose support lattice is the interval $[\widehat{0}, X]$ in $\Lambda$.

4.2. Walks on $\mathbb{R}$-arrangements. Let $\mathcal{A}$ be an essential hyperplane arrangement in $\mathbb{R}^{d}$ with face semilattice $F_{\mathcal{A}}$ and intersection lattice $L_{\mathcal{A}}$. The following is easily seen from observations $(2.1)-(2.5)$. 
Proposition 4.6. $\left(F_{\mathcal{A}}, \circ\right)$ is an LRB semigroup with support lattice $L_{\mathcal{A}}$ and support map span.

Let $C_{\mathcal{A}}$ be the set of regions in the complement of $\mathcal{A}$. There is a one-to-one correspondence $C_{\mathcal{A}} \leftrightarrow \max \left(F_{\mathcal{A}}\right)$. Thus the general theory produces a class of random walks on $C_{\mathcal{A}}$ to which Theorem 4.4 is applicable. The description of this case is as follows.

Random walk on $C_{\mathcal{A}}$ : Fix a probability distribution $w$ on $F_{\mathcal{A}}$. If

the walk is currently in region $C \in C_{\mathcal{A}}$, then choose a face $X \in F_{\mathcal{A}}$ according to $w$ and move to the region $X \circ C$.

Let $P_{w}$ be the transition matrix

$$
P_{w}(C, D)=\sum_{F: F \circ C=D} w_{F}
$$

Theorem 4.4 specializes to the following, where part (3) relies on Zaslavsky's formula (Theorem 2.1) together with relation (4.3).

Theorem 4.7 (Bidigare-Hanlon-Rockmore [2], Brown-Diaconis [15]). .

(1) $P_{w}$ is diagonalizable.

(2) For each $X \in L_{\mathcal{A}}$ there is an eigenvalue $\varepsilon_{X}=\sum_{F: \operatorname{span}(F) \subseteq X} w_{F}$.

(3) The multiplicity of $\varepsilon_{X}$ is $\left|\mu_{L_{\mathcal{A}}}(X, \widehat{1})\right|$.

(4) These are all the eigenvalues.

(5) Assume that the probability mass $w$ is not concentrated on any single hyperplane $H_{i}$. Then there is a unique stationary distribution $\pi$.

Remark 4.8. The following interesting result appears in [3]. Let $w$ be the uniform distribution on the set of vertices (minimal elements of $F_{\mathcal{A}} \backslash\{0\}$ ) of an arrangement in $\mathbb{R}^{3}$. Then the probability (according to $\pi$ ) of being in a region with $k$ sides is proportional to $k-2$. It is an open problem to give any such geometric characterization of the stationary distribution for arrangements in $\mathbb{R}^{d}$, $d \geq 4$.

4.3. Walks on $\mathbb{C}$-arrangements. Let $\mathcal{A}$ be an essential hyperplane arrange-

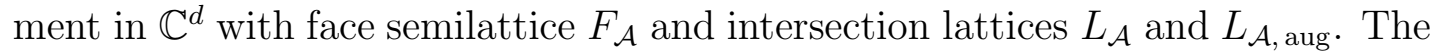
following strengthening of observation (3.2) is immediate.

Proposition 4.9. $\left(F_{\mathcal{A}}, \circ\right)$ is an LRB semigroup with support lattice $L_{\mathcal{A} \text {, aug }}$ and support map span.

Applying the general theory directly to $F_{\mathcal{A}}$ and the ideal $\max \left(F_{\mathcal{A}}\right)$ we get a walk on the maximal complex sign vectors which is a direct analogue of the real walks in Section 4.2 .

Theorem 4.10. The statements of Theorem 4.7 are valid for the complex walks, with the following replacements for items (2) and (3):

(2) For each $X \in L_{\mathcal{A} \text {, aug }}$ such that $\mu_{L_{\mathcal{A} \text {, aug }}}(X, \widehat{1}) \neq 0$ there is an eigenvalue $\varepsilon_{X}=\sum_{F: \operatorname{span}(F) \subseteq X} w_{F}$.

(3) The multiplicity of $\varepsilon_{X}$ is $\left|\mu_{L_{\mathcal{A} \text {, aug }}}(X, \widehat{1})\right|$. 
The proof of part (3) relies here on the generalized Zaslavsky formula (Theorem 5.1) together with relation (4.3). Note that in the formulation of Theorem 4.7 we need not demand that $\mu(X, \widehat{1}) \neq 0$, since that is automatically true for geometric lattices. However, in Theorem 4.10 all we know is that the lattice is lower semimodular, which implies that the Möbius function alternates in sign but not that it is nonzero.

Specializing in various directions there are several semigroup-induced random walks coming out of this situation. We describe two of them.

Case 1. Suppose that the probability mass $w$ is concentrated on the real sign vectors and let $Z=X \circ i Y \in F_{\mathcal{A}}$, for real sign vectors $X$ and $Y$. Choose $W \in F_{\mathcal{A}} \cap\{0,+,-\}^{t}$ according to $w$ and move to $W \circ Z=(W \circ X) \circ i Y$. Then $Z$ and $W \circ Z$ have the same imaginary part $i Y$. It can be checked that the subset of $F_{\mathcal{A}}$ consisting of sign vectors with fixed imaginary part $i Y$ is an LRB semigroup. Note that it doesn't come from a filter of a fiber, as in Proposition 4.5.

For complexified real arrangements, where sign vectors correspond to intervals, we have in this case that

$$
[0, X] \circ[R, S]=[0 \circ R, 0 \circ R \circ X \circ S]=[R, R \circ X \circ S]
$$

So, probability mass concentrated on elements $[0, X]$ (real sign vectors) gives a random walk on the set of intervals $[R, S], S$ maximal, for any fixed element $R$.

Case 2. Let $\mathcal{A}^{\mathbb{C}}$ be the complexification of a real arrangement $\mathcal{A}^{\mathbb{R}}$. We have that $L_{\mathcal{A}^{\mathbb{C}} \text {,aug }} \cong \operatorname{Int}\left(L_{\mathcal{A}^{\mathbb{R}}}\right)$. The purpose here is to determine the transition matrix eigenvalues for the fiber semigroup $\operatorname{Fib}(X)=\left\{y \in F_{\mathcal{A}^{\mathbb{C}}}: \operatorname{supp}(y) \leq X\right\}$, for $X=[\pi, \widehat{1}] \in \operatorname{Int}\left(L_{\mathcal{A}^{\mathbb{R}}}\right)$. The support lattice of $\operatorname{Fib}(X)$ is the interval $[\widehat{0}, X]$ in $L_{\mathcal{A}^{\mathbb{C}}, \text { aug }}$, cf. Proposition 4.5.

Theorem 4.4 shows that the eigenvalues are indexed by intervals $[\alpha, \beta] \in$ $[[\widehat{0}, \widehat{0}],[\pi, \widehat{1}]]$, i.e., intervals $[\alpha, \beta]$ such that $\alpha \leq \pi$. Furthermore, the multiplicity of such eigenvalue is, according to Theorems $4.10,5.1$ and 5.2 , the absolute value of

$$
\mu_{\operatorname{Int}(L)}([\alpha, \beta],[\pi, \widehat{1}])= \begin{cases}\mu_{L}(\alpha, \pi) \mu_{L}(\beta, \widehat{1}), & \text { if } \pi \leq \beta \\ 0, & \text { otherwise }\end{cases}
$$

Thus, eigenvalues of positive multiplicity occur only when $\alpha \leq \pi \leq \beta$, and we have proved the following.

Theorem 4.11. The statements of Theorem 4.4 are valid for the complex hyperplane walks induced on fibers $\mathrm{Fib}(X)$, as explained, with the following replacements for items (2) and (3):

(2) For each $(\alpha, \beta) \in[\widehat{0}, \pi] \times[\pi, \widehat{1}]$ there is an eigenvalue $\varepsilon_{(\alpha, \beta)}$.

(3) The multiplicity of $\varepsilon_{(\alpha, \beta)}$ is $\left|\mu_{L}(\alpha, \pi) \mu_{L}(\beta, \widehat{1})\right|$.

The exact value of $\varepsilon_{(\alpha, \beta)}$ can of course be stated as a special case of Theorem 4.4, but we leave this aside. 
4.4. Walks on libraries. This section concerns the walks produced by the braid arrangements, both real and complex. By translating from permutation and partition structures we can interpret the states of such walks as distributions of books on shelves. This library terminology also provides a convenient image for picturing and explaining these walks.

Real case. Here one obtains random walks on permutations governed by probability distributions $w$ on ordered partitions. This case is thoroughly discussed and exemplified in the literature, see [2, 3, 13, 15, 16]. We mention just two examples.

First, suppose that the probability mass is concentrated on the two-block ordered partitions whose first block is a singleton. That is,

$$
\text { probability }= \begin{cases}w_{i}, & \text { for the partition }\{i\} \mid[n] \backslash\{i\} \\ 0, & \text { for all other ordered partitions }\end{cases}
$$

Then the random walk is precisely the Tsetlin library, for which book $i$ is chosen with probability $w_{i}$ and moved to the begining of the shelf.

Second, more generally allow non-zero probability for all two-block ordered partitions:

$$
\text { probability }=\left\{\begin{array}{l}
w_{E}, \text { for the partition } E \mid[n] \backslash E \\
0, \quad \text { for all other ordered partitions. }
\end{array}\right.
$$

Then the steps of the random walk consist of removing the books belonging to the subset $E$ with probability $w_{E}$ and then replacing them in the induced order at the beginning of the shelf.

In the general case, when non-zero probability is allowed for arbitrary ordered partitions, we obtain the one-shelf dynamic library with several borrowers described in the Introduction.

Complex case. Let us now see what happens in the case of the complex braid arrangement. We work out the case of a particular fiber LRB, namely the one determined by choosing $X=[\pi, \widehat{1}]$, where $\pi$ is a partition $\left(B_{1}, \ldots, B_{k}\right) \in \Pi_{n}$ and $\widehat{1}$ is the partition into singletons.

In our library there are $n$ books labeled by the integers 1 through $n$, and $k$ shelves labeled by the integers 1 through $k$. Think of $\pi$ as a division of the books into $k$ groups corresponding to the blocks $B_{i}$. For instance, $B_{1}$ could be the set of books on combinatorics, $B_{2}$ the set of algebra books, and so on. We are going to consider placements of these $n$ books on the $k$ shelves so that the books in any particular class $B_{i}$ stand (in some order) on some particular shelf dedicated to that class.

The inverse image $\operatorname{supp}^{-1}(X)$ consists of pairs $[p, s]$, where $p$ is an ordered partition of the given blocks, $p=\left\langle B_{p_{1}}, \ldots, B_{p_{k}}\right\rangle$, and $s$ is a permutation of $[n]$ refining $p$. We interpret such an element $[p, s]$ as a particular placement of the books: the books in $B_{p_{1}}$ stand on the top shelf in the order assigned by $s$, then the books in $B_{p_{2}}$ stand on the next shelf in the order assigned by $s$, and so on. 
The fiber semigroup $\operatorname{Fib}(X)=\operatorname{supp}^{-1}\left(\Lambda_{\leq X}\right)$ consists of pairs $[q, t]$, where $q$ is an ordered partition such that $\operatorname{supp}(q)$ is a coarsening of the given partition $\pi=\left\{B_{1}, \ldots, B_{k}\right\}$, and $t$ is an ordered partition refining $q$.

A step in the Markov chain is of the form $[p, s] \mapsto[q, t] \circ[p, s]=[q \circ p, q \circ p \circ t \circ s]$. What is its combinatorial meaning? Well, $q \circ p$ is an ordered partition with blocks $B_{1}, \ldots, B_{k}$, and $q \circ p \circ t \circ s$ is a permutation refining $q \circ p$. Hence, the combinatorial meaning of such a step in the Markov chain is that we permute the shelf assignments for the blocks $B_{i}$ according to $q \circ p$, and then permute the books on each shelf as induced by the permutation $q \circ p \circ t \circ s$.

Here is a concrete example. Say we have 14 books of 4 types, namely the algebra books $B_{\text {alg }}=\{1,4,5,7\}$, the combinatorics books $B_{\text {comb }}=\{2,8,11,12,14\}$, the geometry books $B_{\text {geom }}=\{6,13\}$, and the topology books $B_{\text {top }}=\{3,9,10\}$. Furthermore, say that the present state of the Markov chain is this library configuration:

\begin{tabular}{ccccc}
11 & 14 & 2 & 12 & 8 \\
\hline 6 & 13 & & & \\
\hline 4 & 7 & 5 & 1 & \\
\hline 10 & 9 & 3 & & \\
\hline
\end{tabular}

So, in particular, we have the combinatorics books on the top shelf, the geometry books on the next shelf, and so on.

Now, let

$$
q=\left\langle B_{\text {alg }}\left|B_{\text {comb }} \cup B_{\text {top }}\right| B_{\text {geom }}\right\rangle
$$

and

$$
t=\langle 4,5|1,7| 8,9,12|14| 2,3,10,11 \mid 6,13\rangle
$$

Then, $[q, t]$ acting on the state (4.4) leads to the following configuration

\begin{tabular}{ccccc}
4 & 5 & 7 & 1 & \\
\hline 12 & 8 & 14 & 11 & 2 \\
\hline 9 & 10 & 3 & & \\
\hline 6 & 13 & & &
\end{tabular}

From now on we specialize the discussion to what seems like a "realistic" special case, in which the Markov chain is driven by choices of subsets $E \subseteq[n]$ of the books. This walk has the following description in words.

Library walk: A borrower enters the library and borrows a subset $E \subseteq[n]$ of the books with probability $w_{E}$. These books may come from several shelves. When returned the books are put back in the following way. Permute the shelves so that the ones that contained one of the borrowed books become the top ones, maintaining the induced order among them and among the remaining shelves, which are now at the bottom. Then, on each shelf place the books belonging to $E$ at the beginning of the shelf, in the induced order, followed by the remaining books in their induced order. 
For example, if this procedure is carried out on the library configuration (4.4) for the choice $E=\{1,2,3,4\}$ we obtain the new configuration (4.6).

\begin{tabular}{ccccc}
2 & 11 & 14 & 12 & 8 \\
\hline 4 & 1 & 7 & 5 & \\
\hline 3 & 10 & 9 & \\
\hline 6 & 13 & & \\
\hline
\end{tabular}

In mathematical language, the following is going on. For the subset $E \subseteq[n]$ let $K_{E} \stackrel{\text { def }}{=} \cup_{i: B_{i} \cap E \neq \emptyset} B_{i}$ and

$$
q_{E} \stackrel{\text { def }}{=}\left\langle K_{E} \mid[n] \backslash K_{E}\right\rangle \text { and } t_{E} \stackrel{\text { def }}{=}\left\langle E\left|K_{E} \backslash E\right|[n] \backslash K_{E}\right\rangle \text {. }
$$

The mathematical description of the library walk is that we assign the following distribution

$$
\text { probability }=\left\{\begin{array}{l}
w_{E}, \text { for the partition interval }\left[q_{E}, t_{E}\right], \text { all } E \subseteq[n] \\
0, \quad \text { for all other intervals of ordered partitions }
\end{array}\right.
$$

to the elements of the fiber semigroup $\operatorname{Fib}([\pi, \widehat{1}])$, and then we refer to Theorem 4.11 for the consequences.

To exemplify how the interval $\left[q_{E}, t_{E}\right]$ acts on a library configuration we return once more to the configuration (4.4). Suppose that $E=\{1,2,3,4\}$ and let the interval $\left[q_{E}, t_{E}\right]$ act on (4.4). This leads to the library configuration (4.6), as shown by the following computation table

\begin{tabular}{c||c|c|c|c|c||c|c||c|c|c|c||c|c|c||}
$\circ$ & 11 & 14 & 2 & 12 & 8 & 6 & 13 & 4 & 7 & 5 & 1 & 10 & 9 & 3 \\
\hline \hline $1,2,3,4$ & & & 2 & & & & & 4 & & & 1 & & & 3 \\
\hline $5,7,8,9,10,11,12,14$ & 11 & 14 & & 12 & 8 & & & & 7 & 5 & & 10 & 9 & \\
\hline \hline 6,13 & & & & & & 6 & 13 & & & & & & & \\
\hline \hline
\end{tabular}

Summing up the discussion we obtain the following result.

Theorem 4.12. The statements of Theorem 4.11 are valid for the library walk, with the following replacements for parts (2) and (3):

(2) For each pair of unordered partitions $(\alpha, \beta)$ such that $\alpha \leq \pi \leq \beta$ (i.e., $\beta$ refines $\pi$ and $\pi$ refines $\alpha)$ there is an eigenvalue $\varepsilon_{(\alpha, \beta)}$. Furthermore,

$$
\varepsilon_{(\alpha, \beta)}=\sum w_{E}
$$

the sum extending over all $E \subseteq[n]$ such that $E$ is a union of blocks from $\beta$ and the shelves containing some element of $E$ is a union of blocks from $\alpha$.

(3) The multiplicity of $\varepsilon_{(\alpha, \beta)}$ is $\prod\left(p_{i}-1\right) ! \prod\left(q_{j}-1\right)$ !, where $\left(p_{1}, p_{2}, \ldots\right)$ are the block sizes of $\beta$ and $\left(q_{1}, q_{2}, \ldots\right)$ the block sizes of $\alpha$ modulo $\pi$.

Here part (3) uses the well-known formula for the Möbius function of the partition lattice $\Pi_{n}$ in terms of factorials, see e.g. [20, p. 128] 
Example 4.13. We exemplify the preceding with a worked-out example. Let $n=3$ and $\pi=(1,2 \mid 3)$. Then there are four library configurations indexing the rows and columns of the transition matrix $P_{w}$ :

\begin{tabular}{c|c|c|c|c|} 
& $\frac{12}{3}$ & $\frac{21}{3}$ & $\frac{3}{1} 2$ & $\frac{3}{2} 1$ \\
\hline$\frac{1}{3}$ & $w_{1}+w_{1,2}+w_{1,3}$ & $w_{1}+w_{1,3}$ & $w_{1}+w_{1,2}$ & $w_{1}$ \\
\hline$\frac{2}{3}$ & $w_{2}+w_{2,3}$ & $w_{2}+w_{1,2}+w_{2,3}$ & $w_{2}$ & $w_{2}+w_{1,2}$ \\
\hline$\frac{3}{1} 2$ & $w_{3}$ & 0 & $w_{3}+w_{1,3}$ & $w_{1,3}$ \\
\hline$\frac{3}{2} 1$ & 0 & $w_{3}$ & $w_{2,3}$ & $w_{3}+w_{2,3}$ \\
\hline
\end{tabular}

We ignore the trivial choices $E=\emptyset$ and $E=\{1,2,3\}$, so six elementary probabilities $w_{E}$ are assigned. For instance, the entry $w_{2}+w_{1,2}$ records that if books $E$ are removed from the library configuration $\frac{3}{2} 1$ and replaced according to the rules, then configuration $\frac{21}{3}$ is obtained precisely if $E=\{2\}$ or $E=\{1,2\}$.

We have that $\widehat{0} \triangleleft \pi \triangleleft \widehat{1}(\cdot \triangleleft \cdot$ indicates coverings), so according to Theorem 4.12 there are four pairs $(\alpha, \beta)$ indexing the eigenvalues, all of which have multiplicity one, and these eigenvalues are $\left\{\begin{array}{l}\varepsilon_{(\widehat{0}, \pi)}=0 \\ \varepsilon_{(\widehat{0}, \widehat{1})}=w_{1,3}+w_{2,3} \\ \varepsilon_{(\pi, \pi)}=w_{3}+w_{1,2} \\ \varepsilon_{(\pi, \widehat{1})}=1\end{array}\right.$

It is instructive to also check how the elementary probabilities $w_{E}$ contribute to the various eigenvalues $\varepsilon_{(\alpha, \beta)}$ in terms of the associated intervals:

\begin{tabular}{|c|c|c|}
$E$ & {$\left[q_{E}, t_{E}\right]$} & contributes to $\varepsilon_{(\alpha, \beta)}$ \\
\hline 1 & {$[\langle 12 \mid 3\rangle,\langle 1|2| 3\rangle]$} & {$[\alpha, \beta]=[\pi, \hat{1}]$} \\
2 & {$[\langle 12 \mid 3\rangle,\langle 2|1| 3\rangle]$} & {$[\alpha, \beta]=[\pi, \widehat{1}]$} \\
3 & {$[\langle 3 \mid 12\rangle,\langle 3 \mid 12\rangle]$} & {$[\alpha, \beta]=[\pi, \widehat{1}]$ or $[\pi, \pi]$} \\
1,2 & {$[\langle 12 \mid 3\rangle,\langle 12 \mid 3\rangle]$} & {$[\alpha, \beta]=[\pi, \widehat{1}]$ or $[\pi, \pi]$} \\
1,3 & {$[\langle 123\rangle,\langle 13 \mid 2\rangle]$} & {$[\alpha, \beta]=[\pi, \widehat{1}]$ or $[\widehat{0}, \widehat{1}]$} \\
2,3 & {$[\langle 123\rangle,\langle 23 \mid 1\rangle]$} & {$[\alpha, \beta]=[\pi, \hat{1}]$ or $[\widehat{0}, \widehat{1}]$}
\end{tabular}


4.5. Walks on greedoids. Denote by $E^{*}$ the set of repetition-free words $\alpha=$ $x_{1} x_{2} \ldots x_{k}$ in letters $x_{i} \in E$. A greedoid is a language $\mathcal{L} \subseteq E^{*}$ such that

(G1) if $\alpha \beta \in \mathcal{L}$ then $\alpha \in \mathcal{L}$, for all $\alpha, \beta \in E^{*}$,

(G2) if $\alpha, \beta \in \mathcal{L}$ and $|\alpha|>|\beta|$, then $\alpha$ contains a letter $x$ such that $\beta x \in \mathcal{L}$.

The words in $\mathcal{L}$ are called feasible and the longest feasible words are called basic. All basic words have the same length, and $\mathcal{L}$ is determined by the basic words as the collection of all their prefixes.

Greedoids were introduced in the early 1980s by Korte and Lovász, see the accounts in 12 and [18]. The concept can equivalently be formulated in terms of set systems, but only the (ordered) language version will concern us here.

Important examples of greedoids are provided by matroids (abstraction of linear hull) and antimatroids (abstraction of convex hull). Other examples come from branchings in rooted directed graphs and various optimization procedures (involving some versions of "the greedy algorithm").

If $\alpha, \beta \in \mathcal{L}$ and $|\alpha|>|\beta|$, then repeated use of the exchange property (G2) shows that $\beta$ can be augmented to a word $\beta x_{1} x_{2} \ldots x_{j}$ with $j=|\alpha|-|\beta|$ letters $x_{i}$ drawn from $\alpha$. But the letters $x_{i}$ might not occur in $\beta x_{1} x_{2} \ldots x_{j}$ in the "right" order, i.e., in the order induced by their placement in $\alpha$. This motivates defining an important subclass of greedoids.

Definition 4.14. An interval greedoid is a language $\mathcal{L} \subseteq E^{*}$ satisfying (G1) and the following strong exchange property

(G3) if $\alpha, \beta \in \mathcal{L}$ and $|\alpha|>|\beta|$, then $\alpha$ contains a subword $\gamma$ of length $|\gamma|=$ $|\alpha|-|\beta|$ such that $\alpha \gamma \in \mathcal{L}$.

By subword we mean what can be obtained by erasing some letters and then closing the gaps. Matroids, antimatroids and branchings are examples of interval greedoids.

Let $\mathcal{L}$ be a greedoid on the finite alphabet $E$. We define an equivalence relation on $\mathcal{L}$ by

$$
\alpha \sim \beta \Leftrightarrow\left\{\gamma \in E^{*}: \alpha \gamma \in \mathcal{L}\right\}=\left\{\gamma \in E^{*}: \beta \gamma \in \mathcal{L}\right\} .
$$

So, $\alpha$ and $\beta$ are equivalent if and only if they have the same set of feasible continuations. The equivalence classes $[\alpha] \in \mathcal{L} / \sim$ are the flats of the greedoid, and the poset of flats

$$
\Phi \stackrel{\text { def }}{=}(\mathcal{L} / \sim, \leq)
$$

consists of these classes ordered by

$$
[\alpha] \leq[\beta] \quad \Leftrightarrow \quad \alpha \gamma \sim \beta, \text { for some } \gamma \in E^{*} .
$$

For instance, the poset of flats of a matroid defined in this way is easily seen to be isomorphic to the usual geometric "lattice of flats" of matroid theory.

The feasible words of a greedoid can be composed in the following manner. If $x_{1} x_{2} \ldots x_{j} \in \mathcal{L}$ and $y_{1} y_{2} \ldots y_{k} \in \mathcal{L}$ then

$$
x_{1} x_{2} \ldots x_{j} \circ y_{1} y_{2} \ldots y_{k} \stackrel{\text { def }}{=} x_{1} x_{2} \ldots x_{j} y_{i_{1}} y_{i_{2}} \ldots y_{i_{e}}
$$


where $i_{1}<i_{2}<\ldots<i_{e}$ is the lexicographically first non-extendable increasing sequence such that $x_{1} x_{2} \ldots x_{j} y_{i_{1}} y_{i_{2}} \ldots y_{i_{e}} \in \mathcal{L}$. Letting $\alpha=x_{1} x_{2} \ldots x_{j}$ it is equivalent to say that $\alpha \circ y_{1} y_{2} \ldots y_{k}=\alpha y_{i_{1}} y_{i_{2}} \ldots y_{i_{e}}$ is the word obtained, starting from $\alpha$, by processing the letters $y_{i}$ of $y_{1} y_{2} \ldots y_{k}$ from left to right and adding at the end of the word being formed only those letters $y_{i}$ whose inclusion preserves feasibility.

For instance, consider the greedoid on $E=\{x, y, z, w\}$ whose 14 basic words are the words in $E^{*}$ of length 3 that do not begin with a permutation of $\{x, y, z\}$ or $\{z, w\}$. This greedoid is discussed on pp. 290-291 of [12]. Here are two sample computations:

$$
x \circ y z w=x y w \quad \text { and } \quad(x \circ z) \circ w=x z w \neq x z=x \circ(z \circ w)
$$

This example shows that the composition (4.8) is not associative, and hence does not in general produce a semigroup. For this reason we must limit the discussion to a smaller class of greedoids.

Theorem 4.15. Let $\mathcal{L}$ be an interval greedoid. Then $\mathcal{L}$ with the composition (4.8) is an LRB semigroup. Its support lattice is the lattice of flats $\Phi$, and its support map $\mathcal{L} \rightarrow \Phi$ sends a feasible word $\alpha$ to its class $[\alpha]$.

That matroids give rise to LRB semigroups in this way was mentioned by Brown [13, p.891]. In the matroid case the result is quite obvious, whereas for the general case some details turn out to be a little more tricky. The proof is deferred to Appendix 5.3 .

Being an LRB semigroup means that Brown's theory of random walks, summarized in Section 4.1, is applicable. What can be said about the eigenvalue distribution when specialized to greedoid walks?

There is an eigenvalue $\varepsilon_{X}$ for each $X \in \Phi$ whose value and multiplicity $m_{X}$ are determined according to parts (2) and (3) of Theorem 4.4. However, as Example 4.16 shows, for greedoids the multiplicities do not depend only on the structure of the interval $[X, \widehat{1}]$ in $\Phi$, as was the case in the corresponding situation for real and complex hyperplane walks.

We now illustrate greedoid walks for the important case of branchings. Let $G$ be a directed rooted graph with node set $\{r, 1,2, \ldots, n\}$ and edge set $E$. A branching is a tree directed away from the root node $r$. A subset $R \subseteq\{1,2, \ldots, n\}$ is reachable if it is the set of nodes of some branching.

The branching greedoid $\mathcal{L}_{G}$ consists of ordered strings of edges such that each initial segment is a branching. It models common search procedures on graphs. See [12] and [18] for more information.

The poset of flats of $\mathcal{L}_{G}$ is the lattice $\Phi_{G}$ of reachable sets ordered by inclusion. This is, in fact, a join-distributive lattice, see the cited references. The support map sends a branching to the reachable set of its nodes.

According to Theorem 4.4 there is an eigenvalue $\varepsilon_{X}$ associated with every reachable node set $X$. Its value is the sum of the probabilities for the branchings 
that reach a subset of $X$, and its multiplicity is given by

$$
m_{X}=\sum_{Y: Y \geq X} \mu(X, Y) c_{Y}
$$

Here $c_{X}$ is the number of ordered edge sequences feasibly extending (any branching reaching) $X$ to a maximal branching.

Since $\Phi_{G}$ is join-distributive its Möbius function takes the simple form

$$
\mu(X, Y)= \begin{cases}(-1)^{|Y|-|X|}, & \text { if the interval is Boolean } \\ 0, & \text { otherwise }\end{cases}
$$

For each reachable set $X$, let $\operatorname{dom}(X)$ denote the superset of all nodes that are either in $X$ or else can be reached from $X \cup\{r\}$ along a single edge of $G$. It is clear that every set of nodes contained between $X$ and $\operatorname{dom}(X)$ is reachable, and that the domination set $\operatorname{dom}(X)$ is maximal with this property. Hence, we get the following simplified expression for the eigenvalue multiplicity at $X$ :

$$
m_{X}=\sum_{X \leq Y \leq \operatorname{dom}(X)}(-1)^{|Y|-|X|} c_{Y}
$$

Example 4.16. The rooted directed graph in Figure 4 gives a branching greedoid of rank 3 with 9 basic words: abc, abd, acb, ace, aec, aed, bac, bad, bda. All subsets of $\{1,2,3\}$ except $\{2\}$ are reachable.

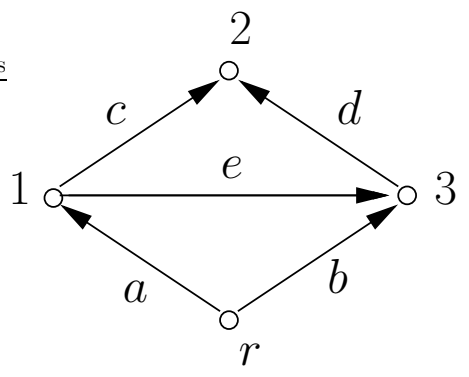

Figure 4. Branching greedoid.

Assign probabilities $w_{\alpha}$ to the seven feasible words (ordered branchings) of rank one and two: $a, b, a b, a c, a e, b a, b d$. A step in the random walk on the nine ordered maximal branchings consists in choosing one of these words $\alpha$ according to the given probabilities $w_{\alpha}$ and then extending $\alpha$ to a maximal branching by adding edges in sequence from the currently visited maximal branching according to the composition rule (4.8).

Here are the eigenvalues for the walk on this branching greedoid: 


\begin{tabular}{c||c|c|c|c|}
$X$ & $c_{X}$ & $\operatorname{dom}(X)$ & $m_{X}$ & $\varepsilon_{X}$ \\
\hline 123 & 1 & 123 & 1 & 1 \\
12 & 2 & 123 & 1 & $w_{a}+w_{a c}$ \\
13 & 2 & 123 & 1 & $w_{a}+w_{b}+w_{a b}+w_{a e}+w_{b a}$ \\
23 & 1 & 123 & 0 & $w_{b}+w_{b d}$ \\
1 & 6 & 123 & 3 & $w_{a}$ \\
3 & 3 & 123 & 1 & $w_{b}$ \\
$\emptyset$ & 9 & 13 & 2 & 0
\end{tabular}

Remark 4.17. By copying the procedure that leads from the sign vector system of a real hyperplane arrangement to that of its complexification (Section 3.3) we can formally introduce the complexification of any LRB semigroup. Namely, let $\Sigma$ be an LRB semigroup with support lattice $\Lambda$. Define $\Sigma^{\mathbb{C}}$ to be the set of intervals $\{[x, y]: x \leq y$ in $\Sigma\}$ with the composition

$$
[x, y][z, w] \stackrel{\text { def }}{=}[x z, x z y w]
$$

One readily verifies that this is an LRB semigroup and that its support lattice is $\operatorname{Int}(\Lambda)$, with the partial order defined in Appendix 5.2.

This way one can complexify e.g. the greedoids walks.

\section{Appendix}

In this section we gather some proofs. Familiarity with the Möbius function is assumed, a good reference is [20].

5.1. A generalized Zaslavsky formula. A ranked poset $R$ with $\widehat{0}$ and $\widehat{1}$ is said to be Eulerian if $\mu_{R}(x, y)=(-1)^{\operatorname{rk}(y)-\operatorname{rk}(x)}$ for all $x<y$ in $R$. Denote by $\max (P)$ the set of maximal elements of a poset $P$.

Theorem 5.1. Suppose that $f: P \rightarrow Q$ satisfies the following conditions:

(1) the posets $P$ and $Q$ are ranked and of the same length $r$,

(2) $Q$ has a unique maximal element $\widehat{1}_{Q}$,

(3) $\widehat{P} \stackrel{\text { def }}{=} P \uplus\left\{\widehat{0}_{P}, \widehat{1}_{P}\right\}$ is Eulerian,

(4) $f$ is an order-preserving, rank-preserving and surjective map,

(5) $\mu_{P}\left(f^{-1}\left(Q_{\leq x}\right)\right)=(-1)^{\mathrm{rk}(x)}$, for all $x \in Q$,

(6) $(-1)^{r-\operatorname{rk}(x)} \mu_{Q}\left(x, \widehat{1}_{Q}\right) \geq 0$, for all $x \in Q$.

Then,

$$
|\max (P)|=\sum_{x \in Q \uplus \widehat{0}}\left|\mu\left(x, \widehat{1}_{Q}\right)\right|
$$

Proof. According to the "Möbius-theoretic Alexander duality" formula [20, p. 137] condition (3) implies that

$$
\mu(R)=(-1)^{r-1} \mu(P \backslash R)
$$

for all subsets $R \subseteq P$. In particular,

$$
|\max (P)|=\mu(\max (P))+1=(-1)^{r-1} \mu(P \backslash \max (P))+1 .
$$


On the other hand, according to the "Möbius-theoretic fiber formula" [21, p. 377] applied to the map $f: P \backslash \max (P) \rightarrow Q \backslash \widehat{1}$ we have that

$$
\mu(P \backslash \max (P))=\mu(Q \backslash \widehat{1})-\sum_{x \in Q \backslash \widehat{1}} \mu\left(f^{-1}\left(Q_{\leq x}\right)\right) \mu\left(x, \widehat{1}_{Q}\right) .
$$

Thus,

$$
\begin{aligned}
|\max (P)| & =1+(-1)^{r-1}\left[\mu(Q \backslash \widehat{1})-\sum_{x \in Q \backslash \widehat{1}} \mu\left(f^{-1}\left(Q_{\leq x}\right)\right) \mu\left(x, \widehat{1}_{Q}\right)\right] \\
& \left.=(-1)^{r} \sum_{x \in Q \uplus \widehat{0}}(-1)^{\mathrm{rk}(x)} \mu\left(x, \widehat{1}_{Q}\right)\right)=\sum_{x \in Q \uplus \widehat{0}}\left|\mu\left(x, \widehat{1}_{Q}\right)\right| .
\end{aligned}
$$

Applying this result to the span map $F_{\mathcal{A}} \rightarrow L_{\mathcal{A}}$ of a real hyperplane arrangement $\mathcal{A}$ we obtain Zaslavsky's theorem 2.1. Applying it to the span map $F_{\mathcal{A}} \rightarrow L_{\mathcal{A} \text {, aug }}$ of a complex hyperplane arrangement $\mathcal{A}$ we obtain Theorem 3.6.

5.2. Lattice of intervals. Let $L$ be a lattice and $\operatorname{Int}(L) \stackrel{\text { def }}{=}\{(x, y): x \leq y\}$ the set of its intervals partially ordered by

$$
(x, y) \leq\left(x^{\prime}, y^{\prime}\right) \quad \text { if and only if } \quad x \leq x^{\prime} \text { and } y \leq y^{\prime} .
$$

The poset $\operatorname{Int}(L)$ is itself a lattice with componentwise operations

$$
(x, y) \vee\left(x^{\prime}, y^{\prime}\right)=\left(x \vee x^{\prime}, y \vee y^{\prime}\right) \text { and }(x, y) \wedge\left(x^{\prime}, y^{\prime}\right)=\left(x \wedge x^{\prime}, y \wedge y^{\prime}\right) .
$$

Its Möbius function is related to that of $L$ in the following way.

\section{Theorem 5.2.}

$$
\mu_{\operatorname{Int}(L)}\left((x, y),\left(x^{\prime}, y^{\prime}\right)\right)=\left\{\begin{array}{lr}
\mu_{L}\left(x, x^{\prime}\right) \mu_{L}\left(y, y^{\prime}\right), & \text { if } x^{\prime} \leq y \\
0, & \text { otherwise } .
\end{array}\right.
$$

Proof. If $x^{\prime} \leq y$ then $\left[(x, y),\left(x^{\prime}, y^{\prime}\right)\right] \cong\left[x, x^{\prime}\right] \times\left[y, y^{\prime}\right]$, so this case follows from the product property of the Möbius function.

Assume that $x^{\prime} \not \leq y$. We claim that the element $\left[x^{\prime} \wedge y, x^{\prime} \vee y\right]$ lacks a latticetheoretic complement in the interval $\left[(x, y),\left(x^{\prime}, y^{\prime}\right)\right]$. For, say that $[s, t]$ is such a complement. This means that

$$
\begin{array}{ll}
s \vee\left(x^{\prime} \wedge y\right)=x^{\prime} & t \vee\left(x^{\prime} \vee y\right)=y^{\prime} \\
s \wedge\left(x^{\prime} \wedge y\right)=x & t \wedge\left(x^{\prime} \vee y\right)=y
\end{array}
$$

Then: $\quad s \leq x^{\prime} \wedge t \leq\left(x^{\prime} \vee y\right) \wedge t=y$

$$
\begin{aligned}
& \Rightarrow s \leq x^{\prime} \wedge y \\
& \Rightarrow s=s \wedge\left(x^{\prime} \wedge y\right)=x \\
& \Rightarrow x^{\prime}=x \vee\left(x^{\prime} \wedge y\right)=x^{\prime} \wedge y \\
& \Rightarrow x^{\prime} \leq y,
\end{aligned}
$$

contradicting the assumption. The interval $\left[(x, y),\left(x^{\prime}, y^{\prime}\right)\right]$ is not complemented, so by Crapo's complementation theorem [20, p. 160] its Möbius function is zero. 
5.3. Interval greedoids. The lattice-theoretical structure of semimodularity is closely related to interval greedoids.

Theorem 5.3. [12, Thm. 8.8.7] The poset of flats $\Phi$ of an interval greedoid is a semimodular lattice. Conversely, every finite semimodular lattice arises from some interval greedoid in this way.

This will be used in the proof of Theorem 4.15, to which we now turn. For economy of presentation we assume familiarity with the notation, conventions and results on pp. $332-334$ of [12]. See particularly the proof of Theorem 8.2.5 on p. 334.

Proof. Let $\alpha=x_{1} \ldots x_{j}$ and $\beta=y_{1} \ldots y_{k}$ be feasible words of an interval greedoid $\mathcal{L}$. By letting $X_{i}=\left[x_{1} \ldots x_{i}\right]$ and $Y_{i}=\left[y_{1} \ldots y_{i}\right]$, these words correspond to edgelabeled unrefinable chains $\emptyset<\cdot X_{1}<\cdot \cdots<\cdot X_{j}$ and $\emptyset<\cdot Y_{1}<\cdot \cdots<\cdot Y_{k}$ in the semimodular lattice $\Phi$. In the same manner (cf. Lemma 8.8.8 of [12]) the composition $x_{1} x_{2} \ldots x_{j} \circ y_{1} y_{2} \ldots y_{k}$ corresponds to the edge-labeled unrefinable chain

$$
\emptyset<X_{1}<\cdots<X_{j} \leq X_{j} \vee Y_{1} \leq \cdots \leq X_{j} \vee Y_{k}
$$

Here, due to semimodularity, the relation $X_{j} \vee Y_{i} \leq X_{j} \vee Y_{i+1}$ is either a covering $X_{j} \vee Y_{i}<X_{j} \vee Y_{i+1}$ or an equality $X_{j} \vee Y_{i}=X_{j} \vee Y_{i+1}$, in which case we omit it from the chain. This shows that

$$
[\alpha \circ \beta]=X_{j} \vee Y_{k}=[\alpha] \vee[\beta]
$$

which in turn is used to see that

$$
[\beta] \leq[\alpha] \quad \Leftrightarrow \quad[\alpha] \vee[\beta]=[\alpha] \quad \Leftrightarrow \quad[\alpha \circ \beta]=[\alpha] \quad \Leftrightarrow \quad \alpha \circ \beta=\alpha
$$

Thus, once associativity of the composition of feasible words has been established the proof will be complete. The other identities required of an LRB semigroup are trivially fulfilled, since feasible words lack repeated letters. Relations (5.3) and (5.4) then show, in view of Proposition 4.3, that $\Phi$ is indeed the support lattice of $\mathcal{L}$ as an LRB semigroup.

To deal with associativity, let $\gamma$ be a third feasible word. We want to show that

$$
(\alpha \circ \beta) \circ \gamma=\alpha \circ(\beta \circ \gamma)
$$

By definition

$$
(\alpha \circ \beta) \circ \gamma=\alpha \beta^{\prime} \gamma^{\prime} \text { and } \alpha \circ(\beta \circ \gamma)=\alpha \beta^{\prime} \gamma^{\prime \prime}
$$

where $\beta^{\prime}$ is a subword of $\beta$ and $\gamma^{\prime}$ and $\gamma^{\prime \prime}$ are subwords of $\gamma$. Thus it remains to convince ourselves that $\gamma^{\prime}=\gamma^{\prime \prime}$. A crucial first step is to show that they are of equal length.

Let $\emptyset<\cdot Z_{1}<\cdot \cdots<\cdot Z_{l}$ be the edge-labelled chain in $\Phi$ corresponding to $\gamma$. Then $(\alpha \circ \beta) \circ \gamma$ corresponds to the chain

$\emptyset<X_{1} \lessdot \cdots<X_{j} \leq X_{j} \vee Y_{1} \leq \cdots \leq X_{j} \vee Y_{k} \leq\left(X_{j} \vee Y_{k}\right) \vee Z_{1} \leq \cdots \leq\left(X_{j} \vee Y_{k}\right) \vee Z_{l}$ 
and $\alpha \circ(\beta \circ \gamma)$ corresponds to

$\emptyset<X_{1}<\cdots<X_{j} \leq X_{j} \vee Y_{1} \leq \cdots \leq X_{j} \vee Y_{k} \leq X_{j} \vee\left(Y_{k} \vee Z_{1}\right) \leq \cdots \leq X_{j} \vee\left(Y_{k} \vee Z_{l}\right)$

Due to associativity of the lattice join operation $\cdot \vee \cdot$ these chains are identical, and by construction the induced edge-labelings yield the words $\alpha \beta^{\prime} \gamma^{\prime}$ and $\alpha \beta^{\prime} \gamma^{\prime \prime}$. Hence, being related to the same segment of the common chain, $\gamma^{\prime}$ and $\gamma^{\prime \prime}$ are of the same length.

We now prove (5.5) by induction on the length of the word $\gamma$. Suppose that $\gamma=t$ is a single letter. Then $\gamma^{\prime}=\gamma^{\prime \prime}$ since the subwords of $t$ of length 0 and 1 are unique. Hence,

$$
(\alpha \circ \beta) \circ t=\alpha \circ(\beta \circ t)
$$

Suppose now that $\gamma=\delta t$, meaning that the last letter of $\gamma$ is $t$. Using the induction assumption and the length one case we obtain

$$
\begin{aligned}
(\alpha \circ \beta) \circ \gamma & =((\alpha \circ \beta) \circ \delta)) \circ t=(\alpha \circ(\beta \circ \delta)) \circ t \\
& =\alpha \circ((\beta \circ \delta) \circ t)=\alpha \circ(\beta \circ(\delta \circ t)) \\
& =\alpha \circ(\beta \circ \gamma) .
\end{aligned}
$$

\section{REFERENCES}

[1] H. Barcelo, and R. Laubenbacher, Perspectives on A-homotopy theory and its applications, Discrete Math. 298 (2005), 36-91.

[2] P. Bidigare, P. Hanlon and D. Rockmore, A combinatorial description of the spectrum for the Tsetlin library and its generalization to hyperplane arrangements, Duke Math J. 99 (1999), $135-174$.

[3] L. J. Billera, K. S. Brown and P. Diaconis, Random walks and plane arrangements in three dimensions, Amer. Math. Soc. 106 (1999), 502-524.

[4] A. Björner, Topological Methods, in "Handbook of Combinatorics" (eds. R. Graham, M. Grötschel and L. Lovász), North-Holland, Amsterdam, 1995, pp. 1819-1872.

[5] A. Björner, letter to R. Laubenbacher, Aug. 7, 2001.

[6] A. Björner, B. Korte and L. Lovász, Homotopy properties of greedoids, Advances in Appl. Math. 6 (1985), 447-494.

[7] A. Björner, M. Las Vergnas, B. Sturmfels, N. White and G. M. Ziegler, Oriented Matroids, Cambridge Univ. Press, 1993. (Second edition 1999)

[8] A. Björner and L. Lovász, Linear decision trees, subspace arrangements and Möbius functions, J. Amer. Math. Soc. 7 (1994), 677-706.

[9] A. Björner, L. Lovász and A. Yao, Linear decision trees: volume estimates and topological bounds, in "Proc. 24th ACM Symp. on Theory of Computing (May 1992)", ACM Press, N.Y., 1992, pp. 170-177.

[10] A. Björner and V. Welker, The homology of "k-equal" manifolds and related partition lattices, Advances in Math. 110 (1995), 277-313.

[11] A. Björner and G. M. Ziegler, Combinatorial stratification of complex arrangements, J. Amer. Math. Soc. 5 (1992), 105-149.

[12] A. Björner and G. M. Ziegler, Introduction to greedoids, in "Matroid Applications" (ed. N. White), Cambridge Univ. Press, 1992, pp. 284-357.

[13] K. S. Brown, Semigroups, rings and Markov chains, J. Theoret. Probab. 13 (2000), 871938 .

[14] K. S. Brown, Semigroup and ring theoretical methods in probability, Fields Inst. Commun. 40 (2004), 3-26. 
[15] K. S. Brown and P. Diaconis, Random walks and hyperplane arrangements, Ann. Probab. 26 (1998), 1813-1854.

[16] P. Diaconis, From shuffling cards to walking around the building: An introduction to modern Markov chain theory, in "Proceedings of the International Congress of Mathematicians, Berlin, 1998", Vol. 1, 187-204.

[17] M. Khovanov, Real $K(\pi, 1)$ arrangements from finite root systems, Math. Res. Lett. 3 (1996), 261-274.

[18] B. Korte, L. Lovász and R. Schrader, Greedoids, Springer-Verlag, Berlin, 1991.

[19] P. Orlik and H. Terao, Arrangements of Hyperplanes, Springer, Berlin, 1992.

[20] R. P. Stanley, Enumerative Combinatorics, Vol. 1, Cambridge Univ. Press, 1997.

[21] J. W. Walker, Homotopy type and Euler characteristic of partially ordered sets, Europ. J. Combinatorics 2 (1981), 373-384.

[22] T. Zaslavsky, Facing up to arrangements: face count formulas for partitions of space by hyperplanes, Mem. Amer. Math, Soc. 1:154 (1976).

Royal Institute of Technology, Department of Mathematics, S-100 44 StockHOLM, SWEDEN

E-mail address: bjorner@math.kth.se 